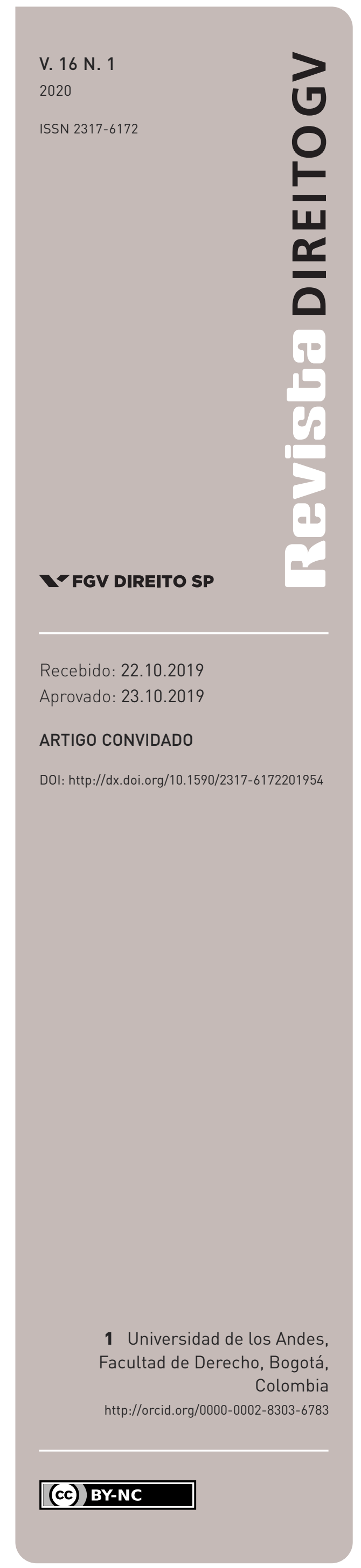

\section{Educación jurídica e innovación tecnológica: un ensayo crítico}

\author{
LEGAL EDUCATION AND TECHNOLOGICAL INNOVATION: A CRITICAL ESSAY \\ EDUCAÇÃO JURÍDICA E INOVAÇÃO TECNOLÓGICA: UM ENSAIO CRÍTICO
}

Daniel Bonilla Maldonado

\section{Resumen}

El artículo se divide en tres partes. En la primera, describo tres de las respuestas más frecuentes a la pregunta: “por qué es necesario, importante o urgente que se incorpore la innovación tecnológica en la educación jurídica?". Las primeras dos respuestas están relacionadas directamente con agentes del mercado que demandan educación jurídica: los estudiantes de derecho y las firmas de abogados. Por un lado, la literatura jurídica que se ocupa de este tema argumenta que las facultades de derecho deben innovar en materia tecnológica para satisfacer las expectativas y necesidades de las nuevas generaciones de estudiantes de derecho. Por el otro, la literatura argumenta que se debe alcanzar este fin para satisfacer las expectativas y necesidades de las firmas de abogados, que son quienes contratan a los nuevos graduandos de las facultades de derecho. La tercera respuesta señala que la innovación tecnológica en las facultades de derecho es necesaria porque permite que los estudiantes alcancen los objetivos de aprendizaje más eficazmente. En la segunda parte, ofrezco mis críticas a las dos primeras respuestas, aquellas que reaccionan y quieren satisfacer las necesidades tanto del mercado de servicios jurídicos como del mercado de servicios educativos. Esta crítica se fundamenta en una lectura heideggeriana de la tecnología. En la tercera parte, presento mis críticas a la tercera respuesta, aquella que conecta estrechamente los fines pedagógicos que persiguen la educación jurídica con la innovación tecnológica. En esta sección del escrito argumento (i) que la tercera respuesta se ve debilitada por la falacia naturalista; (ii) no fundamenta empíricamente sus conclusiones, o no lo hace suficientemente, y presenta algunos de sus argumentos empíricos como absolutos aunque no exista un consenso en la comunidad legal, científica o educativa en torno a ellos; (iii) no ofrece argumentos precisos y detallados que muestren como la innovación tecnológica permite alcanzar los objetivos que usualmente persigue de la educación legal; y (iv) oscurece la relación que existe entre tecnología y poder en la educación jurídica.

\section{Palabras clave}

Educación jurídica; innovación tecnológica; Heidegger; crítica a la tecnología; poder y tecnología.

\section{Abstract}

The article is divided into three parts. In the first part, I describe three of the most frequent answers to the question of why is it necessary, important, or urgent for technological innovation to be incorporated into law schools? The first two answers are directly related to agents of the market that demand legal education: law students and law firms. On the one hand, the legal literature that deals with this issue argues that law schools must innovate in technological matters to meet the expectations and needs of the new generations of law students. On the other hand, the literature argues that this aim should be achieved to satisfy the expectations and needs of law firms, who are the ones that hire new law school graduates. The third response indicates that technological innovation in law schools is necessary because it allows students to achieve learning objectives more effectively. In the second part, I offer a critique of the first two answers, those that react and want to 
meet the needs both of the legal services market and the educational services market. This critique is based on a Heideggerian interpretation of technology. In the third part, I present my critiques of the third answer, which closely connects the pedagogical aims of legal education and technological innovation. In this section of the essay I argue that the third answer (i) is weakened by the naturalistic fallacy; (ii) it does not support empirically its conclusions, or at least does not support them sufficiently, and it presents some of its empirical arguments as absolute, when there is no consensus in the legal, scientific, or pedagogical communities around them; (iii) it does not offer precise and detailed arguments that show how technological innovation may allow us to fulfill the objectives that legal education usually pursues; and (iv) it obscures the connection between technology and power in legal education.

\section{Keywords}

Legal education; technological innovation; Heidegger; criticism of technology; power and technology.

\section{Resumo}

0 artigo está dividido em três partes. Na primeira, descrevo três das respostas mais frequentes à pergunta: "Por que é necessário, importante ou urgente que a inovação tecnológica seja incorporada à educação jurídica?" As duas primeiras respostas estão diretamente relacionadas aos agentes do mercado que exigem educação jurídica: estudantes de Direito e escritórios de advocacia. Por um lado, a literatura jurídica que trata desse assunto argumenta que as faculdades de direito devem inovar em questões tecnológicas para atender às expectativas e necessidades das novas gerações de estudantes de Direito. Por outro lado, a literatura argumenta que esse objetivo deve ser alcançado para atender às expectativas e necessidades dos escritórios de advocacia, que são os que contratam os novos graduados das faculdades de Direito. A terceira resposta indica que a inovação tecnológica nas faculdades de Direito é necessária, pois permite que os alunos alcancem os objetivos de aprendizagem com mais eficiência. Na segunda parte, ofereço minhas críticas às duas primeiras respostas, aquelas que reagem e desejam atender às necessidades do mercado de serviços jurídicos e do mercado de serviços educacionais. Essa crítica é baseada em uma leitura heideggeriana da tecnologia. Na terceira parte, apresento minhas críticas à terceira resposta, a que une estreitamente os objetivos pedagógicos perseguidos pela educação jurídica à inovação tecnológica. Nessa seção do artigo, argumento (i) que a terceira resposta é enfraquecida pela falácia naturalista; (ii) não baseia empiricamente suas conclusões, ou não o faz o suficiente, e apresenta alguns de seus argumentos empíricos como absolutos, embora não exista consenso na comunidade jurídica, científica ou educacional em torno deles; (iii) não oferece argumentos precisos e detalhados que mostram como a inovação tecnológica nos permite alcançar os objetivos que geralmente são perseguidos pela educação jurídica; e (iv) obscurece a relação entre tecnologia e poder na educação jurídica.

\section{Palavras-chave}

Educação jurídica; inovação tecnológica; Heidegger; crítica à tecnologia; poder e tecnologia. 


\section{INTRODUCCIÓN}

La tecnología es un hecho omnipresente e ineludible tanto en la práctica profesional del Derecho como en la educación jurídica contemporáneas. ${ }^{1}$ La tecnología ha impactado de manera notable el mercado de servicios jurídicos en el mundo entero. La práctica jurídica, que hoy tiene dimensiones globales, se ha transformado por la llegada de los abogados en línea, los programas de computador que permiten compartir las pruebas con la contraparte en un juicio (e-discovery), el uso de grandes bases de datos para la toma de decisiones jurídicas, el uso masivo de software que permite crear y compartir documentos jurídicos y el uso de software que permite hacerle seguimiento a los procesos judiciales o hacer comparaciones y análisis de grandes cantidades de documentos jurídicos. Estas innovaciones tecnológicas han logrado que algunos aspectos de los servicios jurídicos sean más eficientes, más transparentes, más baratos y más accesibles a los ciudadanos. ${ }^{2}$ No obstante, estas mismas innovaciones tecnológicas también amenazan con eliminar un número importante de empleos jurídicos, generan riesgos relacionados con la calidad de los servicios legales que se prestan, crean nuevos retos éticos para los abogados y oscurecen o vuelven aun más técnicos aspectos de la práctica jurídica que ahora pasan a ser controlados por algoritmos que solo unos pocos conocen, entienden y pueden transformar. ${ }^{3}$

1 Ver Richard Granat y Marc Lauritsen (2004) (Sobre los desarrollos y posibilidades del E-Lawyering en la práctica jurídica); Stephen M. Johnson (2000); Edward L. Rubin (2012); Brian Sites (2016, p. 21) (Sobre los desarrollos tecnológicos: "Es esencial que los profesores de derecho, como guías de los estudiantes que viven en una era de grandes cambios tecnológicos, se mantengan al día con respecto a los más importantes de estos desarrollos".); Thomas Steele (2003); Richard K. Sherwin, Neal Feigenson y Christina Spiesel (2006, p. 217) ("La práctica del derecho ... depende cada vez más de lo que aparece en las pantallas electrónicas en las salas de audiencia, en las firmas de abogados, en las agencias gubernamentales y en otros lugares. La teoría y la enseñanza del derecho deben también adaptarse a estas nuevas condiciones”.); USC Gould School of Law (2017) ("Si quieren seguir siendo competitivos, los abogados de hoy deben ser capaces de mirar hacia el futuro mientras también comprenden las lecciones del pasado. En el mundo jurídico moderno, esto requiere de un claro entendimiento de cómo la tecnología y la naturaleza omniabarcante del internet han cambiado la cara de la práctica jurídica”.); Miguel Willis (2016) (“La profesión de la abogacía está cambiando rápidamente con el influjo de la tecnología y de los emprendimientos legales”.); Ifeoluwa Olubiyi, Ayobami Olaniyan y Ngozi Odiaka (2015, p. 2) ("La tecnología tiene un papel vital en nuestras rutinas cotidianas y también tiene un impacto significativo en la educación legal y la práctica jurídica”.).

2 Ver Ron Friedman (2004); USC Gould School of Law (2017) ("La práctica de la abogacía virtual tiene una serie de beneficios, tanto para el abogado como para el cliente".); Michele Pistone (2014, p. 590) ("Haciendo uso de las tecnologías contemporáneas, el trabajo que me tomaba 500 horas facturables -casi dos meses en total-puede ser ahora hecho por computadores en unos pocos días".).

3 Ver USC Gould School of Law (2017) (“A pesar de los beneficios el E-Lawyering no es una solución perfecta. Esta nueva estructura también tiene riesgos y desventajas”.); Olubiyi, Olaniyan y Odiaka (2015, p. 7 
Ahora bien, la influencia efectiva y el impacto de estas innovaciones tecnológicas varían dependiendo de las características que tenga la comunidad jurídica y de las peculiaridades que tenga el sector de la innovación tecnológica en cada comunidad política. En América Latina, por ejemplo, las grandes firmas de abogados que pueden pagar por estas innovaciones tecnológicas o que ven que los servicios que prestan a las grandes empresas nacionales o multinacionales se ven afectados por su aplicación ocupan un lugar menor dentro del mercado de servicios jurídicos (BONILLA, 2017, p. 103). La mayoría de los abogados en la región practican el derecho individualmente o en pequeños grupos que comparten gastos de funcionamiento y coordinación, pero no constituyen una persona jurídica autónoma. En Latinoamérica, además, el sector de la innovación tecnológica en materia jurídica no es grande y aunque ha impactado algunas dimensiones de los servicios que prestan las grandes firmas de abogados no ha impactado la práctica de la mayor parte de abogados que ejercen el derecho individualmente, o lo ha hecho solo de manera menor. ${ }^{4}$ En Estados Unidos, en contraste, las grandes firmas de abogados ocupan un lugar notable dentro del mercado de servicios jurídicos ${ }^{\mathbf{5}}$ y el sector de innovación tecnológica en el derecho se ha desarrollado notablemente en los últimos diez años. ${ }^{6}$ Asimismo, estas grandes firmas de abogados tienen el músculo financiero para invertir en tecnología y los servicios que prestan han impactado su práctica, por ejemplo,

y 15); Andrew Beckerman-Rodau (2004, p. 1); Comunidad Virtual, Facultad de Derecho, Universidad Externado (2018) (Argumenta que la tecnología podrá reemplazar a los abogados en el futuro).

4 Ver Daniel Acevedo (2018) (En el 2018 se celebró la primera legaltech en México; es la primera vez en que dos días completos son dedicados a la relación entre tecnología y práctica jurídica); Rodrigo Riquelme (2019) (Las firmas de abogados en América Latina se toman demasiado tiempo para implementar la tecnología); AméricaEconomía.com (s.f.) (Primera reunión Global Legaltech en Ciudad de México).

5 Ver Marc Galanter y William D. Henderson (2007, p. 1869) (“En total, los abogados de las firmas de abogados grandes constituyen el 10.5\% de la profesión jurídica estadounidense. Sin embargo, este es también el sector de la profesión que crece más rápido y es el más próspero y dinámico. Si el pasado reciente es una guía confiable, la institución de las firmas grandes - su poder, influencia y prestigio- serán de nuevo un tema dominante en esta discusión”.); Margaret Grisdela (s.f.).

6 Ver LegalTech hits $\$ 1$ billion investment as lawyers embrace automation (2018), https: / blog.lawgeex.com/ legaltech-hits-1-billion-investment-as-lawyers-embrace-automation/ (visitado por última vez en 9 ago. 2019); Valentin Pivovarov (2019) (2018 fue un año exitoso para la tecnología legal); Olubiyi, Olaniyan y Odiaka (2015, nota 1, p. 4) (Desde los años 80, los computadores han tenido un impacto relevante en la educación e investigación jurídicas); The university map of Legal Tech: who teaches it in the world, THE TECHNOLAWGIST (2019), http://www. thetechnolawgist.com/2019/03/18/the-university-map-of-legaltech-who-teaches-it-in-the-world/ (visitado por última vez en 18 ago. 2019) (Muchas universidades en Estados Unidos tienen proyectos sobre derecho y tecnología.). Ver también, por ejemplo, Pearl Goldman (2001, p. 468); Pearl Goldman (2008) (Lista un gran número de artículos sobre tecnología y derecho, especialmente de Estados Unidos). 
mediante la reducción de las tarifas por hora que pueden cobrar a sus clientes o el número de horas que pueden cobrar por cada servicio que prestan.

La educación jurídica también ha sido impactada por la innovación tecnológica. ${ }^{7}$ En muchas facultades de derecho de todo el mundo se han creado programas macro o micro para la innovación de la enseñanza jurídica mediante la tecnología. En un gran número de facultades de los cinco continentes, herramientas como los cursos en línea, los cursos mixtos (blended), los libros jurídicos electrónicos interactivos, las plataformas en línea para evaluar a los estudiantes, compartir información entre alumnos y profesores, la integración de computadores, teléfonos celulares o contadores (clickers) en las dinámicas de clase, los software antiplagio, el uso de videos en las clases y el uso de software para medir la productividad de los profesores y para evaluar la percepción que tienen los estudiantes de sus cursos tienen una mayor o menor presencia. En consecuencia, las facultades de derecho invierten cada vez más recursos económicos para incorporar o desarrollar estas innovaciones tecnológicas en sus prácticas educativas. Del mismo modo, utilizan estas innovaciones tecnológicas para articular un discurso que les permite presentarse como instituciones educativas de vanguardia y, así, competir más eficientemente en el mercado educativo (LEVY, 2016, p. 251; TENINBAUM, 2019; WILLIS, 2016). ${ }^{8}$ El impacto discursivo o práctico de las innovaciones tecnológicas en la educación jurídica es tan fuerte que las facultades de derecho que no las incorporan son vistas por el mercado (y por ellas mismas) como instituciones desuetas, instituciones que han sido dejadas atrás por el tren de lo "moderno". 9

La innovación tecnológica en la práctica jurídica y en la educación legal, por tanto, ha generado una línea de investigación académica que busca describir sus características, evaluar sus efectos, criticar sus límites y ofrecer horizontes normativos para su implementación o desarrollo adecuado (JOHNSON, 2000; JOHNSON, 2013; LARSON, 2016; LEVY, 2016; MOPPETT, 2013; MURRAY, 2011; OLUBIYI, OLANIYAN y ODIAKA, 2015; PISTONE,

7 Ver, en general, Johnson (2000, p. 101) ("Las facultades de derecho tienen que incrementar, y lo harán, el uso de la tecnología en la enseñanza por una serie de razones".); Steele (2003, p. 614) ("De hecho, la profesión legal y su principal motor de enseñanza, las facultades de derecho, han respondido tanto al rápido cambio tecnológico como a las nuevas exigencias de la sociedad y del público de preparar abogados para que representen adecuadamente a los clientes”.); Sherwin, Feigenson y Spiesel (2006, p. 227); Olubiyi, Olaniyan y Odiaka $(2015$, p. 3) ("La tecnología ha impulsado cambios significativos en la educación y práctica jurídicas en varias jurisdicciones”.).

8 Ver James B. Levy (2016, p. 251) (“... la tecnología tiene un importante simbolismo en las mentes del público y, como la alta costura, transmite un halo de superioridad sobre las escuelas que no la tienen”.).

9 Ver Levy (2016, p. 250-251) ("Los administradores sienten una gran urgencia por adoptar nuevas tecnologías debido a la percepción que tiene el público de que si una escuela no está haciendo precisamente eso, se está quedando atrás...”.). 
2014; STEELE, 2003; SHERWIN, FEIGENSON y SPIESEL, 2006; HARRIS, 2018). Este artículo tiene como objetivo evaluar críticamente tres de las razones más comunes que ofrece la literatura jurídica para explicar o fundamentar las razones por las cuales la innovación tecnológica es necesaria en la educación legal. En consecuencia, el artículo solo se ocupará de la relación entre innovación tecnológica y práctica jurídica de manera tangencial. El texto solo tocará este tema en la medida en que afecte el discurso o las prácticas de las facultades de derecho.

Para cumplir con estos objetivos, divido el artículo en tres partes. En la primera, describiré tres de las respuestas más frecuentes a la pregunta: ¿por qué es necesario, importante o urgente que se incorpore la innovación tecnológica en la educación jurídica? Las primeras dos respuestas están relacionadas directamente con agentes del mercado que demandan educación jurídica: los estudiantes de derecho y las firmas de abogados. Por un lado, la literatura jurídica que se ocupa de este tema argumenta que las facultades de derecho deben innovar en materia tecnológica para satisfacer las expectativas y necesidades de las nuevas generaciones de estudiantes de derecho (JOHNSON, 2000, p. 55; MOPPETT, 2013, p. 102; LARSON, 2016, p. 240; PISTONE, 2014, p. 591; CARON, 2006). ${ }^{10}$ Por el otro, la literatura argumenta que se debe alcanzar este fin para satisfacer las expectativas y necesidades de las firmas de abogados, que son quienes contratan a los nuevos graduandos de las facultades de derecho. ${ }^{11}$ La tercera respuesta señala que la innovación tecnológica en las facultades

Ver Johnson (2000, p. 55) ("Ya que los estilos de aprendizaje de muchos de los estudiantes de las generaciones X yY se han forjado con la ayuda de la tecnología, sería beneficioso incorporar la tecnología de formas más amplias a la pedagogía de las facultades de derecho para responder a los estilos de aprendizaje de esos estudiantes, en lugar de obligarlos a modificar sus estilos de aprendizaje para que quepan en el molde de la pedagogía jurídica tradicional”.); Moppett (2013, p. 102) ("La decisión de usar tecnología para evaluar a los estudiantes no sólo responde a esta expectativa sino que también manda un mensaje a los estudiantes de que sus profesores están comprometidos con su éxito”.); Rogelio Lasso (2002, p. 59) (Sobre las razones por las cuales usar tecnología en la educación legal: "para conectar más adecuadamente con los estudiantes que son el producto de una revolución en el aprendizaje, la tecnología digital también debería integrarse a la enseñanza para así mejorar las experiencias de aprendizaje de los estudiantes sin importar la metodología de enseñanza del profesor ni los estilos de aprendizaje de los estudiantes”.).

11 Ver Pistone (2014, p. 589) (“Las innovaciones tecnológicas también están teniendo repercusiones en la práctica del derecho. Consecuentemente, abogados y estudiantes de derecho deberán desarrollar nuevas habilidades para ser exitosos profesionalmente".); Jeannette Eicks (2012) (“Las firmas necesitan abogados que puedan implementar innovaciones tecnológicas y que puedan entender y facilitar las decisiones apropiadas sobre la tecnología y los proveedores de esta para sus firmas. Un laboratorio de tecnología para la práctica legal generaría una base sólida para los nuevos abogados, al mismo tiempo que se cultivan discusiones más profundas en otras clases”.); Johnson (2000, p. 60-61). Ver, en general, Richard L. Marcus (2008) (Hace referencia a que la tecnología está creando nuevos trabajos que responden a las nuevas necesidades 
de derecho es necesaria porque permite que los estudiantes alcancen los objetivos de aprendizaje más eficazmente (GALVES, 2004, p. 197-198; MOPPETT, 2013, p. 97; LARSON, 2016, p. 238; THREEDY y DEWALD, 2015; WARNER, SOWLE y SADLER, 1998; JOHNSON, 2000, p. 47-48). ${ }^{12}$ En la segunda parte, ofreceré mis críticas a las dos primeras respuestas, aquellas que reaccionan y quieren satisfacer las necesidades tanto del mercado de servicios jurídicos como del mercado de servicios educativos. Esta crítica se fundamenta en una lectura heideggeriana de la tecnología. En la tercera parte, presentaré mis críticas a la tercera respuesta, aquella que conecta estrechamente los fines pedagógicos que persigue la educación jurídica con la innovación tecnológica. En esta sección del escrito argumento que la tercera respuesta (i) se ve debilitada por la falacia naturalista; (ii) no fundamenta empíricamente sus conclusiones, o no lo hace suficientemente, y presenta algunos de sus argumentos empíricos como absolutos aunque no exista un consenso en la comunidad legal, científica o educativa en torno a ellos; (iii) no ofrece argumentos precisos y detallados que muestren como la innovación tecnológica permite alcanzar los objetivos que usualmente persigue de la educación legal; y (iv) oscurece la relación que existe entre tecnología y poder en la educación jurídica.

\section{Mercado, Pedagogía e inNovación TeCnOlógica}

Las relaciones entre innovación tecnológica y educación jurídica se han convertido en un objeto de estudio relevante para los académicos del Derecho del mundo entero (BROUSSARD, 2009; BECKER, n.d.; CARON, 2006; CARON y GELY, 2004; DEGROFF, 2011; COMUNIDAD VIRTUAL, 2018; GALVES, 2004; JOHNSON, 2000; JOHNSON, 2013; KATZ, 2012; LARSON, 2016; LASSO, 2002; LEVY, 2016; MOPPETT, 2013; MURRAY, 2011; OLUBIYI, OLANIYAN y ODIAKA, 2015; PISTONE, 2014; RIQUELME, 2019; RUBIN, 2012; SHERWIN, FEIGESON y SPIESEL, 2006; SITES, 2016; STEELE, 2003;

y expectativas de las firmas de abogados.); Stephanie Kimbro (2010) ("Por lo tanto, [la educación relacionada con la tecnología] debe ser algo que se le ofrezca a los abogados antes de que se sumerjan en la práctica, sea esta privada o dentro de una firma en la que se espera que sigan la política interna sobre el uso de la tecnología.”); Martin J. Katz (2012, p. 823) (“Los que se gradúan de derecho están mal preparados para la práctica”.).

12 Ver Fred Galves (2004, p. 197-198) ("La esencia de la enseñanza es transmitir la información y las ideas de forma tal que puedan ser entendidas por los estudiantes ... los educadores del derecho pueden ofrecerle información a sus estudiantes de una forma efectiva y eficiente si incorporan tecnología visual en sus clases".); Larson (2016, p. 238) ("La tecnología puede ser usada de forma intencional e informada para ayudar y promover el conocimiento o las habilidades que se espera que el estudiante adquiera en cada nivel”.); Debora L. Threedy y Aaron Dewald (2015). Ver también Richard Warner, Stephen D. Sowle y Will Sadler (1998) ("Los computadores son una herramienta efectiva para alcanzar los objetivos pedagógicos que son importantes para la abogacía”.). 
THREEDY y DEWALD, 2015). La descripción y evaluación (típicamente positiva) de las interacciones entre cambios tecnológicos y facultades de derecho (sus discursos y prácticas) se han venido abriendo espacios en la academia del derecho global durante los últimos diez años. Esta literatura tiene una presencia notable en la academia jurídica estadounidense (BROUSSARD, 2009; BECKER, n.d.; CARON, 2006; DEGROFF, 2011; GALVES, 2004; JOHNSON, 2000; JOHNSON, 2013; KATZ, 2012; LARSON, 2016; LASSO, 2002; LEVY, 2016; MOPPETT, 2013; MURRAY, 2011; PISTONE, 2014; RIQUELME, 2019; RUBIN, 2012; SHERWIN, FEIGESON y SPIESEL, 2006; SITES, 2016; STEELE, 2003; THREEDY y DEWALD, 2015). No obstante, también se han abierto algunos espacios en la academia jurídica de otras partes del mundo, entre otras, América Latina y Europa Occidental (CICERO, 2018; COMUNIDAD VIRTUAL, 2018; LATRUP-PEDERSEN, 2002, p. 165-186; MAHARG y MUNTJEWERFF, 2002, p. 307-322). ${ }^{13}$ Una parte de esta literatura se pregunta directamente por la necesidad, urgencia o importancia que tienen las innovaciones tecnológicas en la educación jurídica (MOPPETT, 2013; LASSO, 2002; LARSON, 2016; PISTONE, 2014; OLUBIYI, OLANIYAN y ODIAKA, 2015). Otra parte asume como premisa implícita o explícita las respuestas positivas que ofrecen los académicos del derecho a esta pregunta y escogen como objeto de estudio aspectos más precisos de este campo de estudio, por ejemplo, estrategias para implementar innovaciones tecnológicas particulares como los cursos blended, el uso de contadores (clickers) en las dinámicas de clase o evaluaciones cuantitativas o cualitativas que miden la efectividad de las innovaciones tecnológicas para alcanzar los objetivos de aprendizaje que persiguen las facultades de derecho (BELDARRAIN, 2006, p. 139-153; CARON, 2006; EASTON, 2009; MOPPETT, 2013, p. 106-131; SLOMANSON, 2014). Estos objetivos, típicamente, están directamente relacionados con que los estudiantes reciban y procesen información jurídica, desarrollen destrezas que son necesarias para actuar competentemente en la práctica del derecho y sean conscientes y pongan en práctica los estándares éticos de la profesión (JOHNSON, 2013, n. 31; LARSON, 2016, p. 235-237). ${ }^{14}$

Ahora bien, las respuestas más comunes que ofrece el primer grupo de académicos del derecho, aquellos que se ocupan de responder directamente a la pregunta por la necesidad, pertinencia o urgencia de las innovaciones tecnológicas para la educación jurídica, pueden reunirse en los siguientes tres grupos. El primer grupo de respuestas indica que la innovación tecnológica en la educación jurídica es necesaria, importante o urgente porque solo mediante la incorporación de estas creaciones de la tecnología se puede dar una respuesta satisfactoria

13 Ver, en general, Ámbito Jurídico (2018) (Los estudiantes colombianos deberían comenzar a aprender sobre tecnología). En África, ver Olubiyi, Olaniyan y Odiaka (2015).

14 Johnson (2013, n. 31) (Sobre las diez habilidades fundamentales de los abogados.). 
a las necesidades y expectativas de las nuevas generaciones de estudiantes de derecho. ${ }^{15}$ Esta respuesta, por tanto, parte de dos premisas. Primero, asume que uno de los fines centrales de las facultades de derecho es satisfacer las necesidades y expectativas de los agentes que demandan servicios educativos en el mercado. Segundo, parte de unas descripciones precisas y particulares de estos agentes del mercado. Las caracterizaciones de estos agentes, los estudiantes de derecho, con las que inicia su argumentación este conjunto de respuestas, permiten determinar cuáles son las necesidades y expectativas que las facultades de derecho deberían satisfacer (JOHNSON, 2013, p. 53-55; LASSO, 2002, p. 19-22; LARSON, 2016, p. 232-234; MOPPETT, 2013, p. 98-102). Esta literatura argumenta que la mayoría de los estudiantes de derecho pertenecen a las generaciones $Y$ (los millennials, que se entiende que nacieron entre 1981 y 1995) y Z (los centennials, que se entiende que nacieron entre 1995 y el día de hoy). ${ }^{16}$ La literatura caracteriza a estas generaciones de las siguientes dos formas. En primer lugar, las describe como "nativos digitales". ${ }^{17}$ Para los autores que ofrecen esta descripción, los estudiantes de derecho que pertenecen a estas generaciones nacieron y crecieron con la tecnología. Para estos autores, las generaciones Y y Z, por tanto, son individuos a los que les gusta y que conocen bien la tecnología. Estos estudiantes de derecho, por tanto, "piensan diferente" a otras generaciones y esperan que la tecnología sea parte de los procesos de aprendizaje que tienen lugar en las facultades de derecho. ${ }^{18}$

15 Supra nota 10; ver también Larson (2016) (Sobre la generación Y o millennials: "Ellos están acostumbrados a las ventajas de las tecnologías digitales, y este acceso informa el proceso pedagógico que esperan”.).

16 Johnson (2013, p. 53) ("Por los próximos quince o veinte años, la mayoría de los estudiantes de derecho serán miembros de las generaciones X o Y”.); Moppett (2013, p. 98-99) (“La mayoría de los estudiantes que hoy en día entran a las facultades de derecho son miembros de la Generación Millennial”.). Ver también Murray (2011, p. 194) ("La mayoría de los estudiantes de derecho de hoy en día son miembros de la Generación X y Y [también conocidas como la generación 'millennial']. Los estudiantes millennials serán mayoría entre la población de estudiantes de derecho en los próximos quince a veinte años”.).

17 Ver Moppett (2013, p. 78) ("Los estudiantes que se matriculan hoy en las facultades de derecho son "nativos digitales' - 'nativos en la lengua digital de los computadores, videojuegos e internet”); Johnson (2013, p. 54). Cf. Marc Prensky (2001, p. 1); Larson (2016, p. 232) (“Crecieron rodeados de tecnología. Siempre ha sido parte de su mundo”.).

18 Ver Lasso (2002, p. 19) ("Los estudiantes que ingresan a las facultades de derecho hoy difieren de sus predecesores de hace veinte años porque conocen bien los temas tecnológicos”.); Moppett (2013, p. 100) (Los nativos digitales "no piensan ni procesan material del mismo modo que los miembros de las generaciones previas”.). Ver también Broussard (2009, p. 904) (“Los computadores no cuentan como tecnología para estos nuevos estudiantes, sino que, más bien, están 'instalados en su psique”". [Citando a Jason Frand (2000, p. 16)]; DeGroff (2011, p. 251) (Los estudiantes de derecho de hoy en día "también son muy distintos a las previas generaciones con respecto a la forma en que aprenden y acceden a la información”.). Cf. Prensky (2001, p. 1) ("los estudiantes de hoy piensan y procesan la información de forma fundamentalmente diferente a la de sus predecesores”.). 
En segundo lugar, la literatura describe a las personas que componen estas generaciones como individuos "visuales"; ${ }^{19}$ son individuos que aprenden principalmente a través del sentido de la vista. Estos sujetos, además, aprecian y desarrollan eficazmente varias tareas simultáneamente (multitasking), están interesados en el aprendizaje colaborativo y esperan ser agentes activos, no pasivos, en los procesos de aprendizaje (MOPPETT, 2013, p. 98-101; LASSO, 2002, p. 23; LARSON, 2016, p. 233; JOHNSON, 2013, p. 55; BELDARRAIN, 2006). ${ }^{20}$ Asimismo, estas generaciones esperan ser evaluadas rápidamente y recibir retroalimentación precisa y pronta por parte de sus profesores. Para el grupo de autores que ofrece esta respuesta a la pregunta por la necesidad de la innovación tecnológica en las facultades de derecho, las características de los millennials y los centennials solo se pueden satisfacer eficazmente si la educación jurídica incorpora instrumentos tecnológicos como los computadores, clickers, teléfonos celulares o cámaras digitales en sus dinámicas de clase e incorpora en sus prácticas docentes los distintos tipos de software que permiten el diseño y materialización de cursos en línea, cursos mixtos, la grabación de videos educativos y las plataformas que permiten compartir/editar documentos y evaluar a los estudiantes (SLOMANSON, 2014; BELDARAIN, 2006). ${ }^{21}$

El segundo grupo de respuestas a la pregunta por la necesidad de la innovación tecnológica en la educación jurídica gira en torno a otro de los agentes centrales en el mercado de los servicios educativos: las firmas de abogados. Para este grupo de respuestas, la innovación tecnológica en las facultades de derecho es urgente e importante porque sin ella no sería posible satisfacer las necesidades y expectativas de las firmas de abogados que emplean a las nuevas generaciones de abogados. ${ }^{22}$ Las firmas, se argumenta desde esta perspectiva, indican que la tecnología ha transformado el mercado de servicios jurídicos. Los abogados en línea, el uso de software para comparar y analizar grandes cantidades de documentos jurídicos, el e-discovery, las plataformas que permiten que los ciudadanos y empresas creen y compartan documentos

19 Moppett (2013, p. 100-101) ("Los nativos digitales también tienden a ser estudiantes visuales y quinestésicos que aprenden mejor mediante medios interactivos".); Lasso (2002, p. 29) ("los estudiantes de hoy tienden a aprender de forma visual”.); Johnson (2013, p. 54). Ver también DeGroff (2011, p. 252) (las generaciones X y Y “más probablemente aprenden de manera visual”.); Galves (2004, p. 198) ("Los estudiantes modernos están más acostumbrados a recibir información visual que los estudiantes del pasado”.). Walter Benjamin fue de los primeros que señaló la primacía de la visión en la edad moderna. Ver Benjamin (2014, p. 70-71).

20 Lasso (2002, p. 23) (“Los nuevos estudiantes de derecho aprenden mejor cuando reciben información mediante un medio más dinámico, interactivo y creativo que el texto impreso”.).

21 Supra nota 10.

22 Supra nota 11. 
jurídicos y el uso de grandes bases de datos para la toma de decisiones jurídicas, entre otras herramientas tecnológicas, han transformado la práctica del Derecho. ${ }^{23}$

Por consiguiente, argumenta esta segunda respuesta, las firmas exigen que las facultades de derecho formen a las nuevas generaciones de abogados de manera que puedan satisfacer las necesidades y expectativas que surgen con esta nueva realidad jurídica. Los recién graduados de las facultades de derecho deberían, por tanto, conocer bien las nuevas herramientas tecnológicas que se usan en el mercado de servicios jurídicos y deberían tener la capacidad de contribuir al desarrollo o perfeccionamiento de algunas de estas tecnologías; solo los abogados que puedan manejar estas herramientas sobrevivirán en un mercado jurídico que tiende a reducir la oferta de empleos como consecuencia de la omnipresencia de las innovaciones tecnológicas mencionadas anteriormente. ${ }^{24}$ El trabajo que hacían decenas de abogados jóvenes en las firmas durante varios días o semanas pueden ahora ser realizados en minutos u horas por medio de computadores. Asimismo, los clientes de las firmas de abogados tienen expectativas distintas frente a los servicios que estas prestan: no están ya dispuestos a subsidiar la formación práctica de los abogados jóvenes y esperan pagar menos por el trabajo que realizan las firmas dado que este puede ser adelantado más eficazmente con las nuevas herramientas tecnológicas disponibles en el mercado jurídico. ${ }^{25}$ Las facultades de derecho, por tanto, deberían ofrecer más cursos en donde se enseñe el uso de nuevas tecnologías jurídicas y más cursos que permitan a los estudiantes ser partícipes de los procesos de creación de estas herramientas, por ejemplo, cursos en donde se les enseñe a programar (HARVARD LAW SCHOOL,

23 Ver, en general, John O. McGinnis y Russell G. Pearce (2013, p. 3043); Marcus (2008, p. 264) ("Es decididamente tentador afirmar que la tecnología digital es el principal recurso o al menos un facilitador crítico del papel que tiene el abogado de hoy”.); USC Gould School of Law (2017); Sherwin, Feigenson y Spiesel (2006, p. 227); Jeffrey Allen (2012, p. 4) ("Los abogados deben hacer uso de la tecnología para funcionar exitosamente en el mundo de hoy”.); Pistone (2014, p. 589-591).

24 Ver Pistone (2014, p. 589) (“Las innovaciones tecnológicas también están impactando la práctica del derecho. En consecuencia, los abogados y estudiantes de derecho tendrán que desarrollar nuevas habilidades para ser exitosos profesionalmente. Sin embargo, los clientes corporativos se encuentran con que muchos abogados, incluyendo los recién graduados, carecen de las habilidades relacionadas con los usos más sofisticados de la tecnología relacionada con la abogacía y la práctica jurídica en general”.); Kimbro (2010) ("Evitar el uso de la tecnología en la práctica gerencial no es una opción realista hoy en día”.); Kristin B. Gerdy, Jane H. Wise y Alison Craig (2005, p. 263) ("La naturaleza de la práctica jurídica se está volviendo cada vez más técnica”.); Moppett (2013, p. 103).

25 Ver, en general, Patrick J. Schiltz (1999, p. 899-900) ("El mercado de los servicios jurídicos se ha vuelto cada vez más competitivo ... los clientes insisten en obtener un muy buen trabajo y pagar horas facturables a precios bajos. También insisten en que los abogados minimicen la cantidad de tiempo que le dedican a cada proceso para bajar los precios".); The Irish Times Content Studio (2019) ("los clientes están exigiendo más por menos" [Citando a David Halliwell]). 
2017; SAW, 2018; LOHR, 2017). ${ }^{26}$ De igual forma, otro tipo de materias como las teóricas (Filosofía del Derecho o Filosofía Política, por ejemplo) o interdisciplinarias (Sociología del Derecho, Psicoanálisis del Derecho, Antropología del Derecho, por ejemplo) deberían desaparecer o perder peso en los currículos de las facultades de derecho. ${ }^{27}$ Estas materias, se argumenta, no son útiles o son útiles solo marginalmente para la práctica profesional.

El tercer grupo de respuestas a la pregunta por la necesidad de la innovación tecnológica en las facultades de derecho señala que la tecnología permite alcanzar más eficazmente los objetivos de aprendizaje que estas instituciones persiguen. ${ }^{28}$ En estas instituciones está ampliamente aceptado que dichos fines giran en torno a los siguientes tres pilares: información, destrezas profesionales y competencias éticas (AMERICAN BAR ASSOCIATION, 2014; LARSON, 2016, p. 235-237; MCGINNIS y PEARCE, 2013, p. 3043). ${ }^{29}$ Por un lado, los estudiantes de derecho deben familiarizarse y comprender los contenidos básicos de las principales áreas que componen al ordenamiento jurídico. Igualmente, deben desarrollar las habilidades necesarias para usar adecuadamente los materiales jurídicos y para adelantar las tareas que se requieren para prestar competentemente los servicios que necesitan sus clientes, entre otros, destrezas para entrevistar testigos, habilidades para trabajar en equipo y pericias para redactar distintos tipos de documentos legales como memos, demandas y contratos. Finalmente, los estudiantes de derecho deben conocer, interiorizar y poner en práctica los estándares éticos que regulan la profesión. Deben tener la posibilidad de identificar los conflictos o dilemas éticos que inevitablemente se les presentarán en la práctica y deben tener los conocimientos y herramientas para poder resolverlos conforme a criterios aceptados por la comunidad jurídica a la que pertenecen.

La tecnología, argumentan los autores que presentan este tercer grupo de respuestas, permite alcanzar estos tres objetivos de manera eficaz (LARSON, 2016, p. 238; SLOMANSON,

26 Ver Yenée Saw (2018) ("Los cursos de programación permitirán que los estudiantes de derecho desarrollen las habilidades técnicas para programar y para el diseño de sistemas de información legal”). Ver, en general, Paul Ohm (2009); Jason Morris (2018).

27 Ver James E. Wallace (1967, p. 30) ("La presión cada vez mayor por que haya una educación especializada que prepare a los abogados para las tareas que deben adelantar” [en la práctica profesional, está llevando a que se excluya la filosofía del derecho del currículo de las facultades].); Jan R. Sieckmann (2008) (sobre la marginalización de las disciplinas teóricas, como la sociología jurídica, en las facultades de derecho, especialmente en Alemania).

Supra nota 12 .

29 John O. McGinnis y Russell G. Pearce (2013, p. 3043) (describen “cinco áreas en las que la inteligencia artificial podrá proporcionar servicios ... que actualmente proveen los abogados: revelación de las pruebas entre las partes, investigación jurídica, generación de documentos, generación de expedientes, y predicción de la solución de los casos”.). 
2014; BELDARRAIN, 2006). ${ }^{30}$ Los cursos en línea o mixtos, por ejemplo, permiten que los estudiantes y profesores dediquen más tiempo al desarrollo de habilidades que a la descripción y repetición de contenidos. El uso de computadores, celulares, videos y clickers permiten que los estudiantes estén motivados, atentos y concentrados en clase, lo que contribuye a que puedan procesar mejor la información que se discute o desarrollar las habilidades que se necesitan para manipularla adecuadamente. El uso de libros electrónicos interactivos, para seguir con los ejemplos, permite que los alumnos tengan un papel más activo en los procesos de aprendizaje y el uso de plataformas en línea contribuye a desarrollar habilidades para el trabajo en equipo. Estas plataformas facilitan el trabajo de personas distintas para alcanzar fines comunes como la redacción de documentos necesarios para satisfacer las necesidades de los clientes, la articulación de estrategias de litigio o la formulación de políticas que permitan la búsqueda de nuevos clientes o la retención de los que ya se tienen.

\section{LA TECNOLOGÍA COMO FORMA DE COMPRENSIÓN DEL “SER": UNA CRÍTICA HEIDEGGERIANA A LA TECNOLOGÍA EN LA EDUCACIÓN JURÍDICA}

Los primeros dos grupos de respuestas se fundamentan en una lectura de los abogados, de la práctica profesional y de la educación jurídica que se sustenta en una interpretación de la tecnología como forma de comprensión del "ser". ${ }^{31}$ En esta interpretación, la tecnología no se entiende como un medio para conseguir un fin particular: esta perspectiva sobre la tecnología tampoco se limita a la descripción, análisis o evaluación de una herramienta tecnológica específica, por ejemplo, el Internet, un software particular o una aplicación determinada. La tecnología, más bien, se entiende como el horizonte de perspectivas dentro del cual estamos inmersos. La tecnología se interpreta como la estructura de emplazamiento, ${ }^{\mathbf{3 2}}$ como

30 Ver Larson (2016, p. 238) ("La tecnología... [es] una herramienta que puede ser usada para ayudar a los estudiantes a que alcancen un nivel más alto. La tecnología puede ser usada de forma intencional e informada para apoyar y promover el conocimiento y las habilidades que se esperan que el estudiante obtenga en cada nivel”).

31 Ver, en general, Martin Heidegger (1977) ("La tecnología es un modo de revelar. La tecnología se hace presente [en Occidente] en el ámbito en el que el develar y el desocultar tienen lugar, donde la aletheia, la verdad, suceden”.). Ver también Iain D. Thomson (2005) (“Efectivamente, una vez concebimos la 'esencia' como un verbo y no como un nombre, podemos ver que la 'esencia de la tecnología' hace referencia a la forma en que entidades tecnológicas tienden a 'tematizarse' o venir a nuestra presencia, a suceder para nosotros”.); Hubert Dreyfus (2006, p. 357) (Describe la tecnología como un paradigma cultural nihilista).

32 Heidegger usa la palabra alemana Gestell. Sobre el significado de esta palabra, ver Heidegger (1977, p. 24) (“A aquella interpelación que provoca, que coliga al hombre a solicitar lo que sale de lo oculto como existencia, lo llamamos ahora la estructura de emplazamiento (Gestell)”.); George Steiner (2013, p. 212); Thomson (2005, p. 53). 
la constelación de inteligibilidad que da sentido al "ser" en la modernidad tardía. ${ }^{33}$ La tecnología es el marco desde el cual interpretamos el mundo y los seres humanos. La tecnología es el engranaje, el entretejido de significados, mediante el cual se da cuenta del mundo, de lo que acaece, en la modernidad tardía.

Este horizonte de perspectivas, en la lectura heideggeriana de la tecnología, es el momento más reciente de la genealogía de la metafísica occidental. ${ }^{34}$ La historia de Occidente, para Heidegger, es en lo fundamental la historia de la metafísica; es la historia de las distintas formas de precomprender el mundo que ha construido la filosofía occidental. Hacer una genealogía de la metafísica occidental, por tanto, implica rastrear la emergencia y las transformaciones de las formas de precomprender lo que acaece en el mundo que han sido interiorizadas por Occidente. La estructura de emplazamiento, para Heidegger, emerge con Nietzsche, a pesar de que Nietzsche cuestione radicalmente la tradición metafísica de Occidente y quiera separarse de ella. ${ }^{35}$ Para Heidegger, Nietzsche elabora sin pensarlo una metafísica que sirve como base para la interpretación del "ser" que tiene la modernidad tardía occidental; construye una interpretación de qué es y cómo es ese "ser". Esta estructura de emplazamiento tiene como pilares fundamentales los conceptos de la voluntad de poder y el eterno retorno (THOMSON, 2005, p. 148). El "ser" para Nietzsche, en esta lectura de Heidegger, es una agregación y desagregación infinita de fuerzas que no persigue propósito distinto a su propio crecimiento. El "ser", por consiguiente, se entiende como un recurso ${ }^{36}$ que puede

33 Ver Heidegger (1977, p. 33) ("La cuestión sobre la tecnología es la cuestión sobre la constelación en la que el develarse y el ocultarse, en la que el venir a la presencia de la verdad, toman lugar”.). Ver también Thomson (2005, p. 53) ("En otras palabras, el referente de la frase de Heidegger 'la esencia de la tecnología' es nuestra actual constelación de inteligibilidad histórica, 'la estructura de emplazamiento [Gestell], un modo de develar histórico en el que las cosas se aparecen cada vez más solamente como recursos para ser optimizados".); Dreyfus (2006) (La tecnología como un paradigma cultural que representa y desarrolla nuestro entendimiento tecnológico del "ser", y a partir del cual entendemos lo que es ser una persona, una cosa, un objeto natural, una planta, un animal, etc.).

34 Ver Heidegger (1977, p. 11); Thomson (2005, p. 8-9, n. 5) (Las épocas de la metafísica occidental son la pre-Socrática, la antigua, la medieval, la moderna y, ahora, la de la modernidad tardía).

Ver Thomson (2005, p. 44) (“Heidegger argumenta que la metafísica 'no pensada' de Nietzsche es la responsable de nuestro entendimiento 'tecnológico' nihilista del ser de las entidades y sus consecuencias históricas devastadoras".). Id. en p. 148.

36 Heidegger usa la palabra alemana Bestand. Sobre el significado de Bestand ver, en general, Heidegger (1977, p. 17) (Explica qué implica ser Bestand: "En todas partes todo se ordena que esté a disposición, que esté inmediatamente a la mano, en verdad que esté listo para ser solicitado para un mayor ordenamiento. Lo así solicitado tiene su propio lugar de estancia. Lo llamamos las existencias”.); Thomson (2005, p. 44) (Bestand como "'recursos' listos para ser optimizados, ordenados y aumentados con la mayor eficiencia”.). 
ser ordenado, clasificado y optimizado de manera que pueda ser usado eficientemente. ${ }^{37} \mathrm{El}$ "ser" no tiene un sentido intrínseco. Dios ha muerto; no hay ya una tradición aceptada que pueda dar contenido trascendental al "ser"; el nihilismo es la posición de partida en la modernidad tardía. El "ser", en esta forma de revelamiento, es siempre entendido como una reserva de energía productiva que puede y debe ser usado de manera flexible y efectiva. La estructura de emplazamiento que es la tecnología, en consecuencia, es para Heidegger una forma de desocultar lo oculto, es una forma de revelar el "ser".

Las dos primeras respuestas a la pregunta por la necesidad de la tecnología en la educación jurídica se fundamentan en esta estructura de emplazamiento. En estas respuestas, los estudiantes y los profesores de derecho, así como la educación jurídica, son interpretados como recursos, energía que debe ser optimizada, para satisfacer los intereses del mercado de servicios jurídicos y del mercado de los servicios educativos. Los profesores de derecho y la educación jurídica son recursos para satisfacer tanto las necesidades y expectativas de los estudiantes de derecho como de las firmas de abogados. Asimismo, los estudiantes son entendidos como reservas de energía al servicio de las necesidades y expectativas del mercado de servicios jurídicos. En estas dos respuestas, las herramientas tecnológicas específicas que se promueven son útiles porque permiten poner en operación el proceso de clasificación y optimización de los recursos de energía que son los estudiantes y profesores de derecho. Estos instrumentos tecnológicos particulares permiten materializar la estructura de emplazamiento que domina la modernidad tardía. Los cursos sobre programación, las clases que enseñan a los estudiantes a usar las herramientas que permiten el e-discovery o la comparación y análisis de un gran número de documentos jurídicos, los clickers y los videos, entre otras herramientas tecnológicas particulares que se promueven en la educación jurídica contemporánea, permiten optimizar las reservas de energía que son los estudiantes de derecho. Con el uso de estas tecnologías, los estudiantes de derecho serán fuerzas más eficaces en el mercado de servicios jurídicos, podrán satisfacer más precisa y rápidamente las necesidades y exigencias de los agentes que conforman la oferta y la demanda de servicios jurídicos. Asimismo, estas herramientas tecnológicas (su desarrollo, promoción y uso) interpretan a los profesores de derecho como reservas de energía que pueden optimizarse. Los profesores son recursos que tienen como objetivo optimizar otras reservas de energía, los estudiantes de derecho, para que puedan servir eficazmente al mercado de servicios jurídicos.

El argumento que ofrezco no es que en las dos respuestas que se examinan en este aparte los estudiantes, profesores y la educación jurídica sean valorados únicamente como un

37 Ver Heidegger (1977, p. 16) (Ejemplificando lo que implica ser un recurso: "la energía contenida en la Naturaleza es liberada, lo que es liberado es transformado, lo que es transformado es almacenado, lo que es almacenado es, a su vez, distribuido, y lo que es distribuido es modificado de nuevo”.). 
instrumento del capitalismo; tampoco es una crítica a la mercantilización de la educación jurídica. El argumento que presento es a la vez más preciso y más general: las dos respuestas se fundamentan en una forma de interpretación problemática de los seres humanos y del mundo que habitan que se sustenta en el horizonte de perspectivas tecnológicas que constituye la etapa actual de la historia de la metafísica occidental. Esta interpretación tecnológica del "ser" fundamenta las formas capitalistas de entender la economía. La economía de mercado se construye dentro del entramado de significados tecnológicos dominante. No obstante, este horizonte de perspectivas va mucho más allá de ella; esta forma contingente, no natural, de comprensión del ser atraviesa la interpretación que tenemos de nosotros mismos y del mundo que nos rodea. Por medio de ella, los seres humanos construimos todas las dimensiones que nos constituyen y que constituyen el mundo que habitamos. ${ }^{38}$

Esta estructura de emplazamiento, además, se naturaliza. ${ }^{39}$ Este es su mayor éxito. A pesar de su carácter contingente, se presenta como la única posible. En el caso de la interpretación de la educación jurídica que promueven las dos primeras respuestas anotadas, la mirada que se fundamenta en la forma tecnológica de revelación del "ser" se presenta como la única posible, como la única razonable, como la única que puede efectivamente satisfacer nuestro compromiso con la idea de que los seres humanos y el mundo que nos rodea son fuerzas, energía, recursos que existen para ser ordenados y optimizados. La educación jurídica, por tanto, se profesionaliza radicalmente ${ }^{40}$ se entiende como un medio que permite cuantificar las relaciones cualitativas que existen entre profesores de derecho, estudiantes y

38 Ver Heidegger (1977, p. 24) ("La esencia de la tecnología moderna lleva al hombre por el camino de esa forma de revelar por medio de la cual lo real en todas partes, más o menos inequívocamente, se convierte en una reserva material”.). Ver también Thomson (2005, p. 148) (“El apoyarnos sin pensar en la ontoteología Nietzscheana nos está llevando a transformar todos los seres, incluidos nosotros, en meros 'recursos' [Bestand]; entidades que carecen de significado intrínseco y que son, entonces, despiadadamente optimizadas y ordenadas con máxima eficiencia para servir a los intereses puramente instrumentales de uso flexible”.); David I. Waddington (2005, p. 569) (La tecnología “cambia la forma en que vemos el mundo”); Hubert Dreyfus (2009, p. 27-28) (Todo se vuelve un recurso que puede ser usado y expandido).

39 Ver Heidegger (1977, p. 28) ("El reino de la estructura de emplazamiento amenaza al hombre con la posibilidad de que se le pueda negar la entrada a una forma de revelamiento más original y, por ende, a experimentar la llamada de una verdad más primigenia”). Ver también Waddington (2005, p. 569) ("Heidegger piensa que nuestro estado 'por defecto' es el de estar atrapados en el Gestell”.); Michael Bonnett (1983, p. 22) ("El modo tecnológico de revelar es agresivo en tanto que nos implica en el mundo de una forma en que percibimos el mundo y a nosotros mismos cada vez más únicamente desde su perspectiva, ocultando así y trastocando otras formas de comprender y percibir”.).

Ver Thomson (2005, p. 142) (Explica la profesionalización como resultado de la comprensión tecnológica del ser). 
abogados de firmas. ${ }^{41}$ Todos los agentes involucrados en la educación jurídica se entienden como energía que debe ser optimizada mediante herramientas tecnológicas particulares para que el mercado de servicios jurídicos sea más eficiente en un proceso que solo tiene como objetivo su reproducción infinita.

Estas dos respuestas "tecnológicas" no permiten ver que la educación jurídica puede interpretarse de una manera distinta, aunque para que la interpretación tecnológica de la educación jurídica cambie también debe cambiar el horizonte tecnológico de perspectivas en el que estamos inmersos. El entramado tecnológico oscurece el hecho de que la educación jurídica puede ser un proceso poiético que no convierte en unidimensionales (energía para ser optimizada) a los agentes y relaciones que lo componen. ${ }^{42}$ Este proceso poiético no tiene como objetivo cuantificar y optimizar las relaciones cualitativas entre los estudiantes, profesores y abogados practicantes. Más bien, tiene como fin la construcción de un proceso biunívoco de aprendizaje (estudiantes y profesores) (HEIDEGGER, 1977, p. 5-6) que permita reconocer y comprender la estructura de emplazamiento dominante en la modernidad tardía y la "tecnologización” del abogado que esta genera. Igualmente, se trataría de un proceso biunívoco de aprendizaje que hace explícita y cuestiona la unidimensionalización del abogado que genera la constelación tecnológica de inteligibilidad. Asimismo, sería un proceso poiético que cuestiona la radical profesionalización e hiperespecialización que genera la tecnología como forma de revelación del "ser".

Finalmente, sería un proceso poiético de aprendizaje mutuo entre profesores y estudiantes que busca revelar los posibles significados de la abogacía y el abogado; de revelar sus contenidos. Esta revelación no es una develación de una esencia de la abogacía, de un significado absoluto del abogado. Más bien, es la construcción del abogado como un individuo que puede y debe encarnar las perspectivas normativas históricas que dan sentido a la profesión de la que hacen parte. Las nuevas generaciones de abogados, claro, deben tener la capacidad de servir competente y éticamente a sus clientes. Del mismo modo, deben tener la posibilidad de obtener y mantener trabajos en el mercado de servicios jurídicos. Sin embargo, los abogados no deben entenderse como profesionales que tienen únicamente relaciones contractuales con sus

41 Cf. Heidegger (1977, p. 21) (Explica la forma en que el "ser" es cuantificado en la Naturaleza: "La actitud y el comportamiento ordenadores del hombre se muestran primero en la física moderna que emerge como una ciencia exacta. La forma en que la ciencia moderna representa, persigue y atrapa a la naturaleza es como una calculable coherencia de fuerzas".). Ver Thomson (2005, p. 158) (Explica la cuantificación en la educación: "Este desarrollo ontohistórico explica que la cada vez más ubicua cuantificación de la educación, que preconcibe a los estudiantes como Bestand, no como seres humanos con talentos o capacidades intrínsecas que han ser identificadas y cultivadas, sino más bien como 'resultados' educacionales que han de ser optimizados en términos uniformemente cuantificables".).

42 Ver Heidegger (1977, p. 10) (Sobre el significado de poiesis [лoínoıs] para Heidegger). 
clientes y empleadores, relaciones que tienen como objetivo único satisfacer las necesidades y expectativas de estos grupos de individuos o instituciones. La educación jurídica como proceso poiético debe tener la capacidad de revelar las posibilidades y capacidades de los abogados con respecto a la sociedad y el mundo natural en el que habitan. Esta educación jurídica poiética debe preguntarse y promover formas de entender el discurso y la práctica jurídicos que permitan comprender el papel que debe tener el abogado, por ejemplo, el papel que tiene con respecto al Estado de derecho en el que mora, el reconocimiento y protección de la naturaleza, la materialización de los derechos de los pobres y el desarrollo del pensamiento crítico.

Las herramientas tecnológicas particulares pueden jugar un papel en este proceso poiético, pueden jugar un papel en el proceso de aprendizaje. No obstante, el uso que se les dé dependerá de una reflexión precisa y crítica sobre los objetivos académicos que puede ayudar a alcanzar. Estos objetivos, además, deberán tener en cuenta una interpretación no tecnológica del abogado; una interpretación que le de significado no en abstracto, como energía o recurso, sino en contexto; una interpretación que no pierda de vista al espacio natural y social que habita el abogado. Este abogado, además, debe entenderse cualitativamente, como lleno de contenido a partir de los significados normativos que se han construido históricamente sobre la profesión. La crítica a la tecnología como estructura de emplazamiento desde la cual se interpreta al abogado y a la educación jurídica, por ende, no implica un rechazo absoluto de la tecnología. ${ }^{43}$ La tecnología tiene diversas dimensiones que deben ser apropiadamente descritas y evaluadas. No obstante, la tecnología en la educación jurídica debe interpretarse desde un horizonte de perspectivas no tecnológico. Por consiguiente, debe reconocerse como solo una de las posibilidades por medio de las cuales se interpreta el qué y el cómo del "ser"; debe reconocerse su carácter contingente y parcial como forma de compren-

sión del "ser". ${ }^{44}$ La crítica heideggeriana a la tecnología puede (y debe) entenderse como una

43 Ver Heidegger (1977, p. 15) ("Lo que amenaza al hombre no viene en primer lugar de los efectos posiblemente mortales de las máquinas y los aparatos de la técnica. La auténtica amenaza ha abordado ya al hombre en su esencia. El dominio de la estructura de emplazamiento amenaza con la posibilidad de que al hombre le pueda ser negado entrar en un hacer salir lo oculto más originario, y de que este modo le sea negado experienciar la exhortación de una verdad más inicial. Así pues, donde domina la estructura de emplazamiento, está, en su sentido supremo, el peligro. 'Pero donde está el peligro, crece también lo que salva",.) Este acercamiento se conoce como "unidimensionalismo"; implica la aceptación o el rechazo de la tecnología en su totalidad. Ver Andrew Feenberg (1999, 2000a, p. 237, y 2000b, p. 450). Ver también Thomson (2005, p. 51) (Sobre el "unidimensionalismo”: “...los esencialistas tecnológicos unidimensionalistas deben rechazar o aceptar la tecnología en su totalidad”.).

44 Ver Thomson (2005, p. 68-76) (Sobre la posición de Heidegger en contra del unidimensionalismo: “él no aboga filosóficamente por ninguna forma de rechazo monolítico de la tecnología”.). 
crítica que no unidimensionaliza la tecnología, que no implica tomarla o dejarla como un todo. En la modernidad tardía, la tecnología es inevitable y puede ser valiosa en algunos contextos.

La tecnología puede al mismo tiempo tener usos virtuosos y viciosos. El papel que esta juegue en la vida humana depende del horizonte de perspectivas desde el cual se interprete y se ponga en operación. La tecnología ciertamente puede ocupar un espacio en la educación jurídica. No obstante, este es un espacio limitado que no cambia el campo de juego, como implícita o explícitamente creen los autores que defienden las dos respuestas tecnológicas a la pregunta por la necesidad de la tecnología en la educación legal. La educación jurídica puede ser buena con tecnología o sin tecnología. Igualmente, puede ser mala con o sin tecnología. La fe en que la tecnología cambiará la educación jurídica que subyace a las dos respuestas tecnológicas parece no tener fundamento. Esta fe no es capaz de reconocer el carácter ambiguo que tiene la tecnología, no es capaz de dar cuenta de las dimensiones positivas y negativas que la constituyen, así como tampoco es capaz de reconocer las profundas dificultades que genera el entramado tecnológico de significaciones en el que se sustenta.

La crítica heideggeriana a la tecnología igualmente debe interpretarse como una que reconoce el carácter histórico de la forma tecnológica de revelación del "ser" dominante en la modernidad tardía. ${ }^{45}$ La tecnología no debe interpretarse desde Heidegger como una esencia, como una realidad constituida por componentes invariables que existen desde el inicio de la humanidad. Esta estructura de emplazamiento emerge de manera paradigmática en el siglo XIX con el trabajo de Nietzsche y se desarrolla en el trabajo de otros teóricos que la interpretan y aplican en los siglos XX y XXI. La tecnología es una etapa más en la genealogía de la metafísica occidental; una etapa que, según Heidegger, al mismo tiempo se diferencia y se entronca con las formas de revelación del "ser" presocráticas, antiguas, medievales y de la modernidad temprana. ${ }^{46}$ Todas estas etapas se preguntan por el qué y el cómo del ser; todas estas respuestas ofrecen respuestas que al mismo tiempo precisan aquello que constituye al “ser” y las formas del "ser” originarias de las que devienen todas las demás. 47

45 Ver Feenberg (1999, p. 15) (Sobre el ahistoricismo: "interpreta un fenómeno específicamente histórico en términos de una construcción conceptual transhistórica”.). Ver también Thomson (2005, p. 49) (“En un intento por 'fijar el flujo histórico de la tecnología en una esencia singular', los esencialistas ahistóricos abstraen su comprensión de la esencia de la tecnología del ‘contexto social e históricamente específico' en el que las tecnologías particulares están siempre inmersas”.).

46 Ver supra nota 34.

47 Ver Iain Thomson (2000, p. 298) ("El punto de Heidegger es que al darle forma a nuestra comprensión histórica de 'lo que es' la metafísica termina determinando las presuposiciones más básicas de lo que es cualquier cosa, incluyéndonos a nosotros mismos. 'La humanidad occidental, en todo su comportamiento hacia los seres, e incluso hacia sí misma, es en cada aspecto sostenida y guiada por la metafísica’”.). 
No obstante, todas ellas ofrecen respuestas contingentes a estas preguntas; la cercanía que tenemos con la constelación de inteligibilidad tecnológica generalmente no nos permite reconocerla o entenderla; su cercanía y omnipresencia nos hace interpretarla como un hecho de la naturaleza. Por consiguiente, la educación jurídica tecnológica no debe entenderse como la única forma posible o como la única forma plausible de interpretar la educación legal. Debe entenderse como una interpretación que es consecuencia del dominio de la comprensión de los seres humanos y el mundo que habitan como una reserva de energía, como un recurso abstracto que permite alcanzar un fin determinado. Satisfacer las necesidades y expectativas del mercado de servicios jurídicos y hacer de estudiantes y profesores de derecho recursos óptimos para hacerlo más eficiente son solo algunas de las muchas formas posibles de entender la educación jurídica.

Finalmente, la tecnología como estructura de emplazamiento no debe entenderse como una entidad que existe fuera de los seres humanos, que los controla y frente a la que no se puede hacer nada salvo aceptarla. ${ }^{48}$ En cuanto forma de revelación del "ser" de la modernidad tardía, la tecnología nos constituye; mediante su entramado de significados nos interpretamos e interpretamos el mundo que habitamos. La manera como nos describimos y describimos el mundo está determinada por este horizonte de perspectivas. Cambiar esta estructura de emplazamiento no es nada fácil, implica trabajo y esfuerzo teórico y práctico colectivo de largo plazo. No obstante, no estamos inevitablemente sometidos a la tecnología como forma única de revelación del "ser". En esta interpretación de la tecnología no hay un fatalismo radical. La tecnología no es un agente externo que existe por sí mismo y para sí mismo. Es un aparato metafísico poderoso pero contingente, articulado por seres humanos (THOMSON, 2005, p. 61-68). Por consiguiente, la educación jurídica tecnológica no es tampoco el destino único de la educación legal. Esta forma de darle sentido a la enseñanza del derecho, no obstante, tiene un alto grado de estabilidad. Las constelaciones de inteligibilidad no se transforman de un día para otro. Los procesos y entidades que se basan en ellas, por tanto, también son persistentes y perdurables. Sin embargo, es posible oponerse a la idea de que los estudiantes y profesores de derecho son solo energía sin significado que puede ser optimizada y usada eficientemente, en el caso de la educación jurídica tecnológica, para satisfacer las necesidades y expectativas del mercado de servicios jurídicos o el mercado de servicios educativos (BONNETT, 1983; THOMSON, 2005; FITZSIMONS, 2010; LAMBEIR, 2010). ${ }^{49}$

48 Este acercamiento se llama sustantivismo (fatalismo). Ver Feenberg (1999, p. vii) (Sobre el sustantivismo: "todos están de acuerdo con que la tecnología es una fuerza autónoma separada de la sociedad, una suerte de segunda naturaleza que ejerce una presión sobre la vida social desde el reino separado de la razón”.). Ver también Thomson (2005, p. 49) ("Para el sustantivista, la esencia de la tecnología parece estar moldeando la historia desde afuera, imponiéndose a sí misma como una forma metafísica del más allá que se escapa del todo del control humano".).

Ver Standish (1999, p. 435) (Sobre la discusión crítica de tecnología y educación). 


\section{LA TECNOLOGÍA Y EL APRENDIZAJE EN LA EDUCACIÓN JURÍDICA}

La tercera respuesta que ofrece la literatura jurídica a la pregunta por la necesidad, relevancia o urgencia de la innovación tecnológica en la educación legal tiene cuatro debilidades que se entrecruzan; estas flaquezas argumentativas se articulan y se socavan conjuntamente. Estas debilidades, además, están estrechamente ligadas a las que aquejan a las dos primeras respuestas, aunque algunas de ellas sean propias de esta tercera respuesta. La primera es que aquellos que defienden esta posición derivan el "deber ser" de lo que "es". Los argumentos normativos que defienden se derivan de los argumentos descriptivos que ofrecen, sin que medie ninguna otra razón que permita fundamentar por qué aquello que "es" también "debería ser". La primera debilidad de esta respuesta, por tanto, es que se fundamenta en la falacia naturalista. ${ }^{\mathbf{5 0}}$ Estos autores ofrecen los siguientes tres argumentos: por un lado, hacen una descripción de los millennials y los centennials, que constituyen la mayor parte de los estudiantes de derecho contemporáneos, y una descripción del mercado de servicios jurídicos contemporáneos; por el otro, presentan un argumento normativo que se deriva infundadamente del anterior argumento descriptivo dual: la educación jurídica debe estar al servicio de las necesidades y expectativas que tienen los estudiantes de derecho y de las necesidades y expectativas de los agentes que contratan a estos millennials y centennials cuando se gradúan de las facultades de derecho. Finalmente, argumentan que las innovaciones tecnológicas son necesarias para alcanzar los objetivos que debe perseguir la educación jurídica. La falacia naturalista aparece en el paso del primer al segundo argumento. Del hecho de que los estudiantes de derecho y el mercado de servicios jurídicos tengan ciertas características no puede derivarse sin más que la educación jurídica debe tener como objetivo satisfacer las necesidades y expectativas que surgen como consecuencia de esas características. Tampoco puede derivarse que la tecnología deba ser el medio mediante el cual se satisfagan tales necesidades y expectativas. Este último argumento, sin embargo, será analizado más adelante en este aparte del artículo.

50 Max Black (1964); Stephen Priest (2007, p. 177-178). Formulada inicialmente por David Hume (s.f., p. 245-246): "[e]n todos los sistemas de moral con los que me he topado, he observado que el autor emplea durante cierto tiempo la vía ordinaria de razonamiento, partiendo del ser de Dios o haciendo una serie de observaciones concernientes a los asuntos humanos; cuando de pronto me sorprendo con que la cópula usualmente empleada en las proposiciones, es o no es, es reemplazada en todas las frases por debe o no debe. El cambio es imperceptible, pero tiene una gran trascendencia. Este debe o no debe expresa una nueva relación o afirmación, por lo que resulta necesario poner este hecho de manifiesto y aclararlo. Al mismo tiempo, debe justificarse lo que parece totalmente inconcebible: que esta nueva relación se deduzca de otras que son enteramente distintas". Ver la interpretación de Carlos-Ignacio Massini Correas (1994, p. 114) (“de una serie de afirmaciones acerca de cómo las cosas son, es decir, de proposiciones prescriptivas o especulativas, no puede inferirse -Hume habla de 'deducir'- ninguna aseveración acerca de cómo los hombres deben comportarse, es decir, ninguna proposición práctica”). 
Los siguientes dos ejemplos pueden ayudar a ilustrar y fortalecer esta primera crítica. Los autores que ofrecen esta tercera respuesta describen a los millennials y centennials como individuos que se caracterizan por tener la capacidad de realizar varias tareas simultáneamente (multitasking). ${ }^{\mathbf{5 1}} \mathrm{Al}$ mismo tiempo, señalan que los estudiantes de derecho que pertenecen a estas generaciones evalúan positivamente esta habilidad para adelantar varios trabajos al mismo tiempo. ${ }^{52}$ Los autores que defienden esta tercera respuesta argumentan luego que, como consecuencia de estos hechos, las facultades y profesores de derecho deben incluir en su trabajo docente prácticas que permitan a los estudiantes usar esta capacidad en los procesos de aprendizaje. ${ }^{53}$ La tecnología, argumentan además, sería el mejor medio para alcanzar este objetivo. ${ }^{\mathbf{5 4}}$ Los profesores de derecho deberían entonces incorporar, por ejemplo, el uso de computadores, teléfonos celulares y clickers en sus clases, herramientas tecnológicas que facilitan la realización de varias tareas simultáneamente. Igualmente, los profesores de derecho deberían reconocer el valor y ser pacientes frente a esta práctica que muchos de ellos desconocen, o no desarrollan eficientemente, por pertenecer a una generación distinta a las de sus estudiantes.

El argumento normativo que se ofrece en este ejemplo (deber de promover el multitasking en las prácticas docentes) se deriva del hecho de que los estudiantes de derecho están familiarizados y son buenos para adelantar este tipo de labor. No obstante, no se argumenta por qué es bueno desde el punto de vista académico hacer varias tareas simultáneamente. Por un lado, para ser precisos, lo que se describe como multitasking es realmente la realización secuencial y parcial de varias tareas distintas. Una cosa, por poner un ejemplo coloquial, es

51 Johnson (2013, p. 55) (“estas generaciones son multitaskers ávidos, incluso en el salón de clases”.).

52 Daniel J. Levitin (2014, p. 306) (la ilusión cognitiva, impulsada por un circuito cerrado de adrenalina-dopamina, hace que los multitaskers piensen que están haciendo un mejor trabajo del que están haciendo); Mary L. Courage et al. (2015, p. 6) ("El multitasking promueve una flexibilidad mental que puede realmente cambiar la forma en que aprendemos y retenemos la información, especialmente en el caso de niños pequeños y jóvenes cuya plasticidad neural es relativamente alta”.); Kelvin F. H. Lui y Alan C.-N. Wong (2012, p. 647, 653) ("un mayor grado de multitasking en relación con los medios de comunicación está correlacionado con una mejor integración multisensorial”.). Diane J. Skiba y Amy J. Barton (2006) ([La Generación Net -o las nuevas generaciones- prefieren] "el alfabetismo digital, el aprendizaje experiencial, la interactividad, y la inmediatez”.). Lindsey Farrell y Andrew C. Hurt (2014) (sobre la preferencia de la generación millennial por espacios de trabajo que favorezcan sus habilidades para el multitasking).

53 David Glenn (2010) (algunos profesores defienden que deberíamos incluir el multitasking en nuestras prácticas de enseñanza porque "una de las premisas básicas de una buena pedagogía es que se debe empezar en donde los estudiantes están parados”.).

54 Ver Kristen E. Murray (2011) (emplea datos de encuestas e investigaciones sobre educación para exponer y evaluar los mayores mitos que rodean a los computadores portátiles). Ver también Slomanson (2014). 
caminar y oír música, lo que efectivamente se puede hacer simultáneamente; otra cosa, es leer o contestar algunos correos electrónicos, atender luego la discusión que se está desarrollando en clase, responder posteriormente un mensaje de texto, revisar la actualización de noticias en una página de internet y finalmente tomar algunos apuntes sobre lo que el profesor dijo en la sesión. En este caso no hay ningún tipo de simultaneidad en la realización de las tareas que se adelantan. Por otro lado, este salto del "ser" al "deber ser" desconoce los estudios que muestran que el multitasking es una práctica notablemente ineficiente que reduce los niveles de concentración que son necesarios para realizar tareas complejas y de largo aliento, como muchas de las que deben adelantar los abogados en la práctica profesional. ${ }^{\mathbf{5}}$ Podría ser que haya otros argumentos que fundamentan la idea de que el multitasking es un tipo de práctica que debería ser promovida por las facultades de derecho. No obstante, este tipo de argumento no aparece como parte de la tercera respuesta a la pregunta por la necesidad de la tecnología en la educación jurídica. Esta respuesta tampoco reconoce los estudios que evalúan negativamente la práctica ni ofrece los contrargumentos que responderían a las objeciones que esos estudios presentan en contra de la realización secuencial y parcial de varias tareas por parte de los estudiantes.

El segundo ejemplo está relacionado con el carácter visual de los millennials y centennials. La tercera respuesta argumenta que estas generaciones de estudiantes de derecho aprenden principalmente mediante la vista. ${ }^{\mathbf{5 6}}$ Además, están acostumbrados a hacer uso de materiales visuales como videos, películas y clips de noticias. Por consiguiente, argumenta esta tercera posición, las facultades de derecho deberían promover y privilegiar el uso de materiales audiovisuales en las clases (SLOMANSON, 2014, p. 96-97; GALVES, 2004, p. 198). Creo que sería difícil argumentar que los profesores de derecho no deberíamos incluir tantos materiales didácticos, entre ellos los audiovisuales, como sea posible, si estos permiten alcanzar los objetivos de aprendizaje que perseguimos en nuestras clases. No obstante, en este caso, la argumentación parece perder de vista varios elementos importantes.

Por un lado, que la lectura es también una actividad visual y que la lectura es un elemento central en la actividad de cualquier profesional del derecho. Los abogados tienen como una de sus tareas centrales la lectura de leyes, sentencias, memos, demandas, etc. No es claro, por

55 Ver Interview Clifford Nass (2019) ("Parece ser que los multitaskers son realmente muy malos en todos los aspectos del multitasking. Son terribles para ignorar la información irrelevante; son terribles reteniendo información en sus cabezas de forma ordenada; y son terribles cambiando de una tarea a otra".); Jennifer Lee, Lin Lin y Tip Robertson (2012, p. 104) (“El multitasking interfiere con la adquisición de conocimiento. Genera una carga cognitiva extraña que sobrecarga la memoria. Los estudiantes tienen mejores resultados cuando logran concentrarse en una sola tarea cuando están aprendiendo nuevos materiales dentro y fuera del salón de clases”.). Ver también M. H. Sam Jacobson (2010). 
tanto, por qué las facultades de derecho deberían promover y privilegiar el uso de audiovisuales en las clases por el solo hecho de que los estudiantes de derecho de estas generaciones están acostumbrados y les gustan los audiovisuales. El argumento único o central que ofrece la respuesta tres es que las facultades de derecho deberían satisfacer las expectativas o necesidades que generan las características que definen las identidades generacionales de los estudiantes. Un claro ejemplo, creo, de la falacia naturalista.

No obstante, se podría decir que realmente lo que se quiere argumentar en la respuesta tres es que los profesores de derecho deberían reducir el número de lecturas y aumentar el número de audiovisuales en sus clases. Sin embargo, la literatura que ofrece la respuesta tres, típicamente, no ofrece argumentos precisos que digan cuál es el balance ideal entre lecturas y audiovisuales y cómo se fundamentaría en términos pedagógicos. La respuesta tres guarda silencio frente a preguntas que debería enfrentar como las siguientes: ¿Cuáles son los objetivos de aprendizaje que los audiovisuales permiten alcanzar y cuáles no? ¿Si tanto los audiovisuales como las lecturas son materiales que se perciben mediante la vista, por qué debería privilegiarse a los primeros? ¿La supuesta motivación que genera entre los estudiantes la interacción con audiovisuales justifica por sí misma privilegiar dichos materiales en clase? ¿El costo de oportunidad de no interactuar con otros materiales o desarrollar otras habilidades está justificado?

Sin embargo, la respuesta tres no parece tener en cuenta que la educación jurídica, en parte, tiene como objetivo transformar algunos elementos que caracterizan a los estudiantes. ${ }^{57} \mathrm{La}$ educación jurídica, en ocasiones, debe tener como fin transformar la realidad de los educandos. Los estudiantes, por ejemplo, pueden tener pocas habilidades interpersonales, ser individualistas o no tener disciplina para adelantar proyectos de largo aliento. Los estudiantes, además, pueden sentirse cómodos con estas características que conforman sus identidades y no tener ningún interés en transformarlas. Creo, sin embargo, que pocas facultades de derecho argumentarían que la educación jurídica no debería intentar cambiar o moderar estas características. ¿No sería deseable que los estudiantes de derecho desarrollen habilidades para interactuar con otras personas, habilidades que son necesarias para servir competentemente a sus clientes? ¿No sería deseable que los estudiantes de derecho aprendan a trabajar en equipo y a colaborar con otros para el desarrollo de sus actividades laborales? ¿No sería deseable que los estudiantes de derecho desarrollen habilidades relacionadas con la concentración por periodos más largos que los que exigen los videos y las redes sociales? Como argumenté anteriormente, del "ser" no se puede derivar el "deber ser" sin razones

57 Ver Md Esahaque Sk (2017, p. 1) ("La educación se considera el instrumento más poderoso y efectivo del cambio y del desarrollo sociales en nuestro tiempo. El cambio social implica un cambio en la estructura social y en la función de varias de las unidades que forman la sociedad”.); Saikat Chakraborty et. al. (2018) (sobre la educación como la causa principal del cambio social, cultural e individual -su propósito es transformar la perspectiva y la actitud de las personas y de la sociedad; cambiar la forma en que los estudiantes piensan, etc.). 
externas que fundamenten por qué lo que "es", “debe ser". Asimismo, lo que "es" no siempre está justificado moral, política o pedagógicamente. En estos casos, el fin de la educación debería ser la transformación de lo que "es".

La segunda debilidad de la tercera respuesta está relacionada con el tercer paso argumentativo que la constituye y que mencioné arriba: la tecnología es una herramienta necesaria para alcanzar los objetivos de aprendizaje que buscan materializar las facultades de derecho. ${ }^{\mathbf{5 8}}$ Sin innovación tecnológica, se argumenta, no podrían alcanzarse estos objetivos en la medida en que no tienen en cuenta quienes "son" los estudiantes de derecho. La tecnología se presenta entonces como el medio ineludible al que tienen que apelar las facultades de derecho para satisfacer las necesidades y expectativas de los estudiantes y así poder cumplir eficientemente con las metas de aprendizaje que persiguen. Una parte importante de la literatura que ofrece la respuesta tres no fundamenta sus argumentos empíricamente o no lo hace de manera completa y suficiente. Una parte importante de esta literatura, además, no interactúa con la literatura que proviene de las facultades de educación o las facultades de medicina que son centrales para describir, comprender y evaluar los hechos que interpreta. ${ }^{\mathbf{5 9}}$

La literatura sobre educación ofrece argumentos precisos relacionados con los objetivos de aprendizaje que deberían perseguirse, los medios para alcanzarlos, su eficiencia, los efectos no buscados que generan, entre otras cosas. ${ }^{60}$ La literatura médica ofrece, por ejemplo, argumentos relacionados con la manera como los seres humanos aprendemos, la relación entre nuestros sentidos, el cerebro y el mundo que queremos aprehender, los efectos que genera en nuestro cerebro y en nuestros procesos de aprendizaje la interacción con distintos tipos de estímulos externos o el papel que juegan la memoria o los genes en estos procesos (LEVY, 2016, p. 255-276; GALLAGHER, 2001, p. 146, 163; RATEY, 2001, p. 185-195; SHEEL et al., 2010, p. 13, 22, 25-26; SYLWESTER, 1995; MEDINA, 2011; KAHNEMAN, 2011; FOER, 2011). ${ }^{61}$ En un campo interdisciplinario como el de la pedagogía, el cerebro, siempre serán más exitosas que las aproximaciones que se basan en observaciones sobre los hábitos cambiantes de los estudiantes en relación con la tecnología”.); ver Shahid Alvi (2011, p. 136-137). Ver también infra notas 59 y 60 .

Douglas Kellner (2000); John Palfrey (2012, p. 122-123) (los profesores de derecho deben incorporar las investigaciones de otros campos para informar su pedagogía en lugar de apoyarse en el comportamiento observable de los estudiantes); Peter C. Brown et al. (2014, p. 106-108); Lawrence A. Cunningham (2012, p. 95-96, 100); Maryanne Wolf (2007, p. 70-71); Larry Cuban (1986, p. 3, 19, 27, 72-73); Roger Schank (2011, p. xv, xvi, 13, 16, 207-209) (los educadores deben entender cómo funciona el cerebro).

Ver Robert Sylwester (1995) (sobre cómo la intuición acerca de cómo aprenden los estudiantes lleva a errores, sobre-generalizaciones y estereotipos). 
parecería que la literatura jurídica que se ocupa de la relación entre tecnología y educación jurídica debería conocer y usar esta literatura; los argumentos que ofrece la respuesta tres se sustentan, en muchos casos, en presunciones sobre temas que la literatura médica y sobre educación ya han fundamentado apropiadamente o han desvirtuado con argumentos persuasivos. ${ }^{62}$ No es un secreto que buena parte de los profesores de derecho en todo el mundo son autodidactas en cuestiones pedagógicas. ${ }^{63}$ Hemos aprendido haciendo; hemos aprendido mediante un proceso de ensayo y error que generalmente ha ignorado los argumentos técnicos sobre la pedagogía y los procesos neurológicos por medio de los cuales los seres humanos conocen el mundo.

En ocasiones, la literatura que ofrece la respuesta tres presenta información empírica para sustentar sus argumentos. No obstante, presenta esta argumentación como no controversial, como absoluta, como conclusiones frente a las cuales hay consenso en la comunidad académica. ${ }^{64}$ Sin embargo, una parte importante de los argumentos que sustentan las caracterizaciones de los estudiantes de derecho o de la relación entre tecnología y procesos de aprendizaje en los que se basa la respuesta tres son profundamente controversiales. Dos ejemplos pueden ayudar a ilustrar y justificar mi argumento. El primero es la caracterización de las generaciones Y y Z que fundamenta la respuesta tres. Esta caracterización presenta a estas generaciones como entidades monolíticas; ${ }^{65}$ todos los individuos nacidos entre 1980 y 1995 o entre 1995 y hoy en día comparten ciertas características que son centrales para su identidad, entre otras, son individuos visuales y nativos digitales.

62 Levy (2016, p. 252) (sobre el espiral de complacencia que surge entre los autores y sus supuestos sobre los nativos digitales). Daniel Haun (2011, p. 293) (sobre cómo las personas tienden a confundir la repetición con la verdad, debido al efecto de la 'ilusión de verdad' - los supuestos de los nativos digitales se entienden como demasiado evidentes para ser cuestionados); Bennet et al. (2008, p. 775, 779, 780, 783); S. Bennett y K. Maton (2010, p. 328); Chris Jones y Binhui Shao (2011, p. 2, 34).

63 Levy $(2016$, p. 251) (Explica que los profesores tienden a usar la tecnología de forma intuitiva, debido a la presión que sienten por innovar.); Slomanson (2014, p. 93).

64 Ver Sue Bennett et al. (2008, p. 779) (aunque muchas aseveraciones sobre los nativos digitales apelan al sentido común, ellas carecen de cualquier forma de sustento empírico).

65 Ver Bennett y Maton (2008, p. 324) (sobre cómo los nativos digitales son supuestamente distintos: la mayoría de ellos no tienen habilidades precisas relacionadas con el Internet, aunque algunos de ellos conozcan, entre otras, las herramientas del Internet 2.0 o las que permiten crear contenido); Lauren Troksa (2016) (sobre cómo "los nombres tienen poder" y las generaciones han sido tradicionalmente nombradas - para dar un título, una identidad o una definición- de maneras que pueden ser engañosas, tergiversadas, equivocadas. Los millennials, Baby Boomers, etc., son representados como grupos radicalmente diferentes de personas que son, dentro del grupo, homogéneas. Estas dos premisas se alejan radicalmente de la verdad). 
Estas generalizaciones, además, parten de estudios más o menos precisos o rigurosos que se realizan en países específicos, usualmente, del Norte Global. ${ }^{66}$ No obstante, las conclusiones de estos estudios se generalizan a todo el globo. ${ }^{67}$ Las conclusiones más o menos precisas o rigurosas con respecto a la parte se extienden al todo. Estas generalizaciones resultan problemáticas porque homogenizan un campo que es profundamente heterogéneo. ¿Realmente todos los estudiantes de derecho de Estados Unidos tienen las características que se le atribuyen a las generaciones $Y$ y Z ¿ ¿Las variables de clase, raza y género no relativizan o desvirtúan estas generalizaciones? ¿Una joven negra y pobre que proviene de una zona rural en Lousiana tiene la misma relación con lo visual y el multitasking que un joven blanco de clase media que fue criado en Nueva York? Aun más problemático, ¿un joven keniata "Y" de clase media, una joven vietnamita "Y" de clase baja y un joven colombiano "Y" de clase alta tienen la misma relación con el aprendizaje colaborativo o comparten la idea de que los estudiantes deben tener un papel activo en los procos de aprendizaje? Esta última pregunta es particularmente importante en contextos en donde la educación jurídica es multicultural, como Estados Unidos, México o Alemania. No parece acertado asumir que todos los estudiantes de derecho que llegan a las facultades de estos países tienen la misma relación con la tecnología o con los procesos de enseñanza. ${ }^{68}$ Asumir ciegamente las caracterizaciones de las generaciones puede llevar a cometer errores en la manera como se diseñan y aplican los cursos de derecho multiculturales.

Ahora bien, una lectura más caritativa del argumento diría que las generalizaciones sobre los estudiantes de derecho Y y Z son útiles, aunque imprecisas, en la medida en que permiten describir tendencias culturales que afectan la manera cómo las nuevas generaciones construyen sus identidades o sus contenidos. Esta interpretación, creo, puede ser cierta. No obstante, una cosa es utilizar estas generalizaciones para describir procesos culturales macro y otra muy distinta es usar esas generalizaciones para derivar conclusiones precisas sobre cómo

66 Por ejemplo, el término millennial fue acogido por Neil Howe y William Strauss (1991), haciendo clara y exclusiva referencia a una generación de jóvenes en Estados Unidos. Desde entonces, es un término que se ha extendido a todos los jóvenes del globo. Ver también Marc Prensky (2001).

67 Ver supra nota 65; Jones y Shao (2011, p. 34) ("Más que mostrar que hay una Net Generation de nativos digitales que son naturalmente competentes en materia tecnológica, la evidencia empírica de una serie de países muestra que las experiencias de los estudiantes con las tecnologías están lejos de ser universales”.) (Se debe aclarar que incluso estos estudios son usualmente desarrollados en Estados Unidos).

68 Nicole R. Zarrett y Oksana Malanchuk (2005) (sobre cómo la decisión de los jóvenes de adelantar una carrera en temas relacionados con la tecnología y cualquier forma de conocimiento relacionado con tecnología depende del género y de la raza); Kellner (2002) en general (sobre la división digital entre los que tienen y los que carecen de tecnología, especialmente sobre la manera como esta división se materializa en el salón de clase y en la educación en general). 
se debería enseñar derecho, cómo aprenden los seres humanos y dónde deberían invertirse los recursos escasos que están a disposición de las facultades de derecho, ¿en tecnología o en la contratación de nuevos profesores, la compra de libros para la biblioteca o la creación o fortalecimiento de un programa de becas para estudiantes de bajos recursos económicos?, por ejemplo. No muchas facultades de derecho en el mundo tienen los recursos económicos para financiar todos estos proyectos al mismo tiempo.

El segundo ejemplo es la caracterización de las generaciones Y y Z como compuestas por nativos digitales que, por esta razón, piensan diferente a otras generaciones. ${ }^{69}$ Por un lado, el argumento parecería impreciso. El hecho de que la tecnología esté presente en la vida cotidiana de los jóvenes que pertenecen a las generaciones $\mathrm{Y}$ y $\mathrm{Z}$ no significa que la conozcan bien o que la puedan usar eficientemente. Múltiples estudios muestran que el uso que hacen los jóvenes de la tecnología se concentra fundamentalmente en las redes sociales, los mensajes de texto y plataformas de juegos y videos. Los jóvenes de estas generaciones ciertamente han crecido alrededor e interactuando con computadores. No obstante, no hay información empírica que muestre que estas generaciones conozcan bien y manejen eficazmente programas básicos como Word, Excel y PowerPoint; tampoco que tengan nociones básicas de programación. ${ }^{70} \mathrm{Si}$ esto es cierto, no quedaría claro en qué sentido esta familiaridad general con algunas tecnologías podría ser útil para la enseñanza del derecho, tampoco cómo las tecnologías que efectivamente conocen estas generaciones deberían incluirse urgente e ineludiblemente en las clases de derecho o qué objetivos pedagógicos podrían contribuir a lograr.

Por el otro lado, aun más cuestionable es el argumento que se deriva de la familiaridad de las generaciones Y y Z con la tecnología, esto es, que estos jóvenes "piensan distinto"71 a otras

70 Bennett y Maton (2010, p. 321, 324) ("De hecho, con la excepción de las redes sociales, la mayoría de las actividades asociadas con la Web 2.0 son realizadas por una minoría de los que responden estas encuestas de gran escala"; ellos no crean contenido, programan muy poco y, más bien, usan el Internet, sobre todo, para sus actividades sociales); Jones y Shao (2011, p. 34) ("Aunque sí hubo un crecimiento considerable en el acceso de los estudiantes universitarios a tecnologías computacionales y herramientas tecnológicas en línea, en algunos contextos su uso de las tecnologías se destinó principalmente a propósitos sociales y de entretenimiento, no al aprendizaje”.); Penny Thompson (2013, p. 20, 23) (sobre las encuestas que demuestran que los estudiantes universitarios tienen una competencia muy limitada y solo con respecto a algunos aparatos que se usan para un rango pequeño de actividades como socializar, jugar, y navegar la red); Shiang-Kwei Wang et al. (2014, p. 643 , 655) (investigaciones sobre estudiantes de bachillerato muestran que no son más competentes con la tecnología de lo que son sus profesores); Megan O’Neil (2014) (propone que la aseveración de que los estudiantes de hoy son más competentes tecnológicamente es un mito).

71 Bennett et al. (2008, p. 783) ("las nuevas investigaciones retan la noción de una generación homogénea con experticia técnica y un estilo de aprendizaje particular”.); Bennett y Maton (2010, p. 328); Mark Bullen et al. (2011, p. 17-18); Chris Jones et al. (2010, p. 731). 
generaciones y, por tanto, que la educación jurídica debe adaptarse a las nuevas formas de pensar que han desarrollado los seres humanos de finales del siglo XX e inicios del XXI. Si tomamos en serio el argumento, y no solo lo interpretamos como una exageración retórica, este parecería no tener fundamento. La literatura médica no reconoce que el cerebro de las nuevas generaciones haya cambiado o que los procesos neurológicos que permiten el aprendizaje sean distintos. ${ }^{\mathbf{7 2}}$ Estos cambios estructurales, de formas de pensar, solo se dan en procesos evolutivos de muy largo plazo - procesos que se miden en siglos y no en décadas. ${ }^{\mathbf{7 3}}$ Actualmente, no existe información empírica que sustente que, por su familiaridad con la tecnología, los estudiantes de derecho "piensen distinto” a los profesores. Esto no quiere decir, claro, que sean idénticos.

El uso de tecnología como los teléfonos celulares, el correo electrónico, las redes sociales, etc., ha cambiado algunos patrones de conducta individuales o sociales: los miembros de las nuevas generaciones que tienen familiaridad con la tecnología, por ejemplo, tienen mayores dificultades para concentrarse por periodos extensos, esperan respuestas inmediatas a sus comunicaciones y recogen información de forma distinta y más eficazmente que otras generaciones mediante instrumentos digitales (GALLAGHER, 2010, p. 152; LEVITIN, 2014, p. 96; RAVIZZA et al., 2014; RICHTEL, 2012; PINKER, 1997; DAVIS, 2009, p. 33 40). No es claro, sin embargo, cuáles de estas transformaciones, si alguna, deberían tenerse en cuenta para modificar la educación jurídica o cómo la tecnología podría contribuir a potenciar sus efectos positivos y neutralizar los negativos. Es posible argumentar que sería deseable que las facultades de derecho tuvieran en cuenta estas transformaciones individuales y sociales. No obstante, para fundamentar este argumento normativo, habría que describirlas más precisamente, examinar de manera detallada sus causas y consecuencias y explorar los posibles Maton (2010, p. 328); Bullen et al. (2011, p. 1, 17-18) ("no hay evidencia de que los estudiantes llamados nativos digitales tengan necesidades pedagógicas diferentes”.); Ellen J. Helsper y Rebecca Eynon (2010, p. 517-518) (sobre que no hay sustento empírico para hacer una distinción entre los "nativos digitales" y los "inmigrantes digitales"; aún más, dicha distinción puede terminar siendo prejudicial para su educación); Matt Richtel (2012, p. A18) (la tecnología puede estar cambiando los comportamientos relacionados con el aprendizaje de los estudiantes, pero ningún estudio de largo plazo justifica que esté cambiando sus ciclos de atención); Mark Pagel (2011, p. 70) (basándose en la biología evolutiva, Pagel concluye que sabemos que el Internet no ha cambiado el cerebro humano porque las personas que no tienen acceso a él piensan igual a las que sí tienen acceso); Steven Pinker (2011, p. 86, 87); Gregory Paul (2011, p. 122) (la única forma de saber si el Internet está cambiando la forma en que pensamos y aprendemos es mediante un experimento simulado, y no queda para nada claro cómo haríamos algo así). Levy (2016, p. 244) ("de forma significativa, los expertos nos dicen que es muy poco probable que el cerebro sea siquiera capaz de realizar los cambios sugeridos por aquellos que aseguran la existencia de la tal división cognitiva”.).

Roger Schank (2019) (“El Internet no ha cambiado la forma en que pienso ni la forma en que nadie piensa”.). 
impactos negativos o positivos que podrían tener para los procesos de aprendizaje que se adelantan en las facultades de derecho.

La tercera debilidad, estrechamente conectada con las dos anteriores, es que la respuesta tres no basa sus conclusiones en reflexiones precisas y detalladas sobre la relación entre tecnología y educación jurídica. Es poco común encontrar que esta literatura presente argumentos puntuales sobre la manera cómo los objetivos de aprendizaje que persiguen las facultades de derecho (familiaridad y uso de información legal, desarrollo de destrezas profesionales y creación de competencias éticas) se conectan negativa o positivamente con tecnologías específicas. No es común encontrar en esta literatura argumentos concretos fundamentados empíricamente que permitan entender, por ejemplo, cómo los libros electrónicos interactivos, las plataformas para compartir y editar información, los clickers y los teléfonos celulares contribuyen a lograr los fines pedagógicos que usualmente persiguen las facultades de derecho. Muchos de estos autores asumen acríticamente que estas tecnologías efectivamente ayudan a alcanzar tales fines o presentan argumentos anecdóticos, intuiciones o estudios de caso puntuales que no permiten llegar a conclusiones generales sobre los objetos de estudio que examinan. La información disponible no permite concluir que la tecnología ha cambiado (o podría cambiar) el campo de juego, la estructura, de la educación jurídica. Para que sea realmente persuasiva, la respuesta tres debería precisar los vínculos que tienen o podrían tener la educación jurídica (sus fines, medios y agentes) y la tecnología. Asimismo, debería alejarse de una promoción radical y acrítica de la tecnología que la presenta como indudablemente positiva para las facultades de derecho.

Finalmente, la cuarta crítica a la respuesta tres, que se conecta de manera clara con la crítica heideggeriana a la tecnología que usé en el primer aparte del ensayo, cuestiona uno de los supuestos generales en los que se fundamenta esta respuesta: la tecnología es un medio neutral para alcanzar los fines que usualmente persigue la educación jurídica. Con Heidegger, se puede hacer explícito que la tecnología no es un mero instrumento neutral para alcanzar distintos tipos de fines individuales o sociales. ${ }^{\mathbf{7 4}}$ Con Heidegger, es posible ver que la tecnología en la modernidad tardía es más bien una forma mediante la cual se revela el "ser". La tecnología, desde esta perspectiva, es la constelación de inteligibilidad contingente que da sentido a los seres humanos y al mundo que habitamos. ${ }^{75}$ En esta parte final del escrito, no obstante, quisiera examinar otra debilidad del argumento que entiende la tecnología como un medio neutral. Este supuesto oscurece el carácter ambiguo de la tecnología, oscurece que la tecnología puede tener al mismo tiempo efectos esperados y no esperados, positivos y

74 Heidegger (1977, p. 12) (“La tecnología no es entonces un mero medio. La tecnología es una forma de revelar”.). 
negativos en tanto que emerge e interactúa con estructuras de poder que pueden generar efectos distributivos problemáticos. La tecnología, su creación y uso, no surge en el vacío; surge siempre en contextos específicos en donde las relaciones de poder determinan quiénes tienen o no tienen acceso a ella, así como la distribución de sus consecuencias negativas y positivas. ${ }^{\mathbf{7 6}}$

Tres ejemplos me pueden ayudar a ilustrar y fundamentar el argumento. El primer ejemplo hace explícitos los nexos entre clase social y tecnología. La clase social a la que se pertenece determina el acceso, familiaridad y capacidad de uso de la tecnología (KELLNER, 2000, p. 247-249; EBO, 1998). La tecnología no se distribuye igualitariamente de manera natural entre todas las clases sociales. Un joven de la periferia bogotana, por ejemplo, no tiene el mismo acceso o familiaridad con la tecnología que un joven de la clase media alta de Bogotá o un joven que proviene de una zona rural del departamento de Putumayo, en el sur de Colombia. Los tres, sin embargo, pueden hacer parte de la misma facultad de derecho pública o privada. En consecuencia, la defensa acrítica de la premisa que entiende la tecnología como un medio neutral puede contribuir a la perpetuación de las desigualdades de clase que deberían eliminarse o al menos moderarse mediante la educación. El uso de la tecnología que promueve la respuesta tres no reconoce ni reflexiona sobre el impacto que tendría la tecnología en las aulas compuestas por individuos de distintas clases sociales.

Los estudiantes de bajos recursos, por ejemplo, no necesariamente tienen acceso a computadores portátiles en su vida cotidiana, no necesariamente manejan programas básicos como Word, Excel o PowerPoint o no necesariamente manejan herramientas como los clickers o las plataformas para editar documentos conjuntamente. El uso acrítico de la tecnología en las aulas de clase puede poner en desventaja a los estudiantes no privilegiados económicamente. No hay duda de que estos estudiantes podrían aprender a usar la tecnología que se use en clase. No obstante, este hecho les impondría una carga indebida que se suma a las cargas académicas que todos los estudiantes deben asumir. No es claro, sin embargo, que los estudiantes de bajos recursos puedan acceder a la tecnología; por más que se esfuercen no podrían comprar las herramientas tecnológicas que se les exige en clase para poder cumplir con los objetivos de aprendizaje que se persiguen en las clases de derecho.

76 Kellner (2000, en general, y p. 248): "He estado desarrollando lo que he llamado una teoría crítica de la tecnología que critica los usos o tipos de tecnología que sirven como herramientas de dominación, que rechaza la primacía y las pretensiones de las nuevas tecnologías, que ve las limitaciones de la pedagogía y de las propuestas de enseñanza que se basan principalmente en la tecnología y que no hacen un adecuado énfasis en la pedagogía, en el empoderamiento de los profesores y de los estudiantes, que insiste en llevar a cabo una reforma pedagógica y una restructuración de las estructuras de enseñanza para promover una democracia multicultural, y que promueve una reestructuración apropiada de la tecnología para servir a la sociedad y a la educación democrática de hoy”. 
El segundo ejemplo examina los llamados que hacen algunos de los autores que ofrecen la respuesta tres a que las facultades de derecho incluyan clases de programación dentro de los currículos. ${ }^{77}$ El objetivo de estos cursos no es, generalmente, que los abogados se conviertan en programadores expertos. Más bien, su fin es que los abogados aprendan los conceptos básicos de la programación y puedan adelantar ejercicios básicos de programación. Cuando estas propuestas se presentan acríticamente como medios neutrales para alcanzar fines valiosos, como formar abogados que tengan la capacidad de interactuar con los clientes de las muchas compañías tecnológicas que requieren de servicios jurídicos de calidad, pierden de vista la manera como estos objetos de estudio se entrecruzan con las desigualdades de género. Existe una amplia literatura que muestra como las niñas y mujeres jóvenes no se interesan o no acceden fácilmente a los espacios que constituyen las disciplinas académicas relacionadas con la computación (ZARRETT y MALANCHUK, 2005; WARREN y BOURQUE, 1991; MOGHADDAM, s.f.). Históricamente, estas disciplinas han sido un espacio fundamentalmente masculino.

La promoción acrítica de estos cursos en las facultades de derecho puede convertirse en un mecanismo que reproduce las desigualdades entre los estudiantes de derecho hombres y mujeres. Las estudiantes probablemente no estarán interesadas en este tipo de clases por la relación histórica que ha habido entre género y ciencias de la computación o, aunque estén interesadas, probablemente no tendrán los mismos conocimientos o familiaridad con la programación que sus pares masculinos. Como consecuencia, las estudiantes de derecho tendrán acceso a menos espacios académicos que sus pares masculinos o tendrán algunas desventajas para tener el mismo éxito en estos espacios académicos. Asimismo, una vez se gradúen, los estudiantes tendrán ventajas en el mercado laboral que las estudiantes no tendrán: aquellos tendrán mayores oportunidades para interactuar con un segmento de la demanda de servicios jurídicos que es particularmente poderoso económica y políticamente.

Finalmente, el tercer ejemplo hace evidente que la adopción y el uso de la tecnología compiten por recursos escasos con otro tipo de sujetos u objetos que se requieren para el adecuado funcionamiento de una facultad de derecho, por ejemplo, libros para la bibliote$\mathrm{ca}$, becas para estudiantes de bajos recursos económicos, más profesores de planta y mejoras en la infraestructura de las instituciones. ${ }^{78}$ Escoger la tecnología sobre las otras posibles áreas de inversión de los recursos escasos de los que disponen las facultades de derecho implica una decisión política que jerarquiza las distintas necesidades o aspiraciones que tienen estas unidades académicas. La tecnología en este caso no es un medio neutral en la medida en que

77 Supra nota 26.

78 Olubiyi, Olaniyan y Odiaka (2015, p. 17) ("Los altos costos de la tecnología son un impedimento importante para su uso”.). 
exige no invertir en otras áreas pedagógicamente relevantes de la facultad. Asumir acríticamente la premisa de que la tecnología es un agente neutral oscurece este hecho y dificulta su examen y crítica por parte de los distintos agentes que componen una facultad de derecho. Esta tensión puede ser menor en facultades del Norte Global que tienen muchos recursos para invertir en la satisfacción de sus necesidades y anhelos académicos. En estos casos, puede que todas las necesidades y anhelos puedan satisfacerse razonablemente al mismo tiempo. Sin embargo, en las facultades de derecho del Sur Global, usualmente este no es el caso. Los recursos disponibles son mucho menores y permiten satisfacer algunas necesidades o anhelos y no otros, algunos de ellos en niveles muy menores. La tecnología no es, por tanto, un medio neutral que puede y debe implementarse de manera inmediata, urgente y necesaria en todas las facultades de derecho.

\section{CONCLUSIONES}

La tecnología es un hecho inevitable y omnipresente en la práctica y la educación jurídicas de la modernidad tardía. La tecnología ha cambiado o está cambiando la práctica del derecho en todo el globo. El mercado de los servicios jurídicos, por ende, exige que la educación jurídica también se transforme. La educación jurídica, argumenta una parte notable de la literatura jurídica especializada, debe reformarse, debe adecuarse a los cambios que ha sufrido la práctica profesional. Asimismo, esta literatura argumenta que la educación jurídica debe adecuarse a las necesidades y exigencias de las nuevas generaciones de estudiantes de derecho. Las facultades de derecho deben adaptarse a las características de las generaciones Y y $\mathrm{Z}$ que constituyen la mayor parte de la demanda del mercado de servicios educativos contemporáneos. La educación jurídica, además, debe utilizar la tecnología para alcanzar estos fines. La tecnología es un instrumento necesario y urgente para satisfacer las demandas de los principales agentes que conforman los mercados de servicios jurídicos y educativos.

Esta literatura, sin embargo, pierde de vista las profundas debilidades que tiene la estructura de emplazamiento en la que se fundamenta; pierde de vista los límites que tiene la tecnología como constelación de inteligibilidad dominante en la modernidad tardía. Desde esta perspectiva, los estudiantes y profesores de derecho, los abogados y firmas, así como las facultades de derecho, son meros recursos sin contenido que deben ser optimizados para su uso eficiente. Igualmente, parte de esta literatura pierde de vista la relación entre tecnología y poder; ignora la literatura médica y sobre educación que es necesaria para describir, comprender y evaluar las relaciones entre tecnología y educación jurídica; fundamenta algunos de sus argumentos centrales en supuestos empíricos dudosos; y no siempre conecta de manera precisa tecnologías específicas con los objetivos de aprendizaje que persiguen las facultades de derecho.

La tecnología puede jugar un papel valioso en la educación jurídica. No obstante, este papel solo puede materializarse mediante un reconocimiento, comprensión y transformación del 
entramado de significación dominante que le ha dado sentido históricamente; solo puede materializarse si examina la relación entre poder y tecnología y reconoce que este es un campo ineludiblemente interdisciplinario.

\section{AGRADECIMIENTOS}

El autor agradece a Gabriela Pedraza y Andrés Jurado el magnífico trabajo que realizaron como asistentes de investigación.

\section{REFERENCIAS}

ACEVEDO, Daniel. Tecnología, Derecho y la transformación de esta profesión: ¿por dónde empezar? (I), ÁMBITO JURÍDICO (2018), https: / /www.ambitojuridico.com/noticias/columnista-online/tic/ tecnologia-derecho-y-la-transformacion-de-esta-profesion-por-donde (visitado por última vez en 8 ago., 2019).

ALLEN, Jeffrey. Technology for the Mobile Lawyer in All of Us, 29 GPSOLO 4 (2012).

ALVI, Shahid. Proceed with Caution:Technology Fetishism and the Millennial Generation, 8 INTERACTIVE TECH. \& SMART EDUC. 135 (2011).

ÁMBITO JURÍDICO. Educación y tecnología: de la mano (2018), https: / /www.ambitojuridico.com/ noticias/tecnologia/educacion-y-cultura/educacion-y tecnologia-de-la-mano (visitado por última vez en 9 ago. 2019).

AMÉRICAECONOMÍA.COM. Global Legal Tech Venture Day llega a México para buscar y apoyar nuevas empresas, https: / / mba.americaeconomia.com/articulos/notas/global-legal-tech-ventureday-llegamexico-para-buscar-y-apoyar-nuevas-empresas (visitado por última vez en 8 ago. 2019).

AMERICAN BAR ASSOCIATION, A.B.A. Report and Recommendations, TASK FORCE ONTHE FUTURE OF LEGAL EDUC. 1, disponible en https:/ /www.americanbar.org/content/dam/aba/administrative / professional_responsibility/report_and_recommendations_of_aba_task_force.pdf (2014).

BECKER, David M. Some Concerns About the Future of Legal Education, 51 J. LEGAL EDUC. 469 (s.f.). 
BECKERMAN-RODAU, Andrew. Ethical Risks from the Use of Technology, 31 RUTGERS COMPUTER \& TECH. L.J. 1 (2004).

BELDARRAIN, Yoany. Distance Education Trends: Integrating new technologies to foster student interaction and collaboration, ROUTLEDGE, v. 27, n. 2 (ago. 2006).

BENNETT, Sue et al. The 'Digital Natives' Debate: A Critical Review of the Evidence, 39 BRIT. J. EDUC. TECH. (2008).

BENNETT, S.; MATON, K. Beyond the 'Digital Natives' Debate: Towards a More Nuanced Understanding of Students'Technology Experiences, 26 JOURNAL OF COMPUTER ASSISTED LEARNING (2010).

BENJAMIN, Walter. Baudelaire. José Manuel Cuesta Abad (ed.), Alfredo Brotons Muñoz et al. trans., 2014.

BLACK, Max. The Gap Between "Is" and “Should”, 73 THE PHILOSOPHICAL REVIEW 165 (1964).

BONNETT, Michael. Education in a Destitute Time, 17 JOURNAL OF PHILOSOPHY OF EDUCATION 21 (1983).

BONILLA, Daniel. Los Mandarines del Derecho: El trabajo jurídico pro bono en perspectiva comparada, en Los Mandarines del Derecho: Transplantes jurídicos, análisis cultural del Derecho y trabajo pro bono SIGLO DEL HOMBRE EDITORES, UNIVERSIDAD DE LOS ANDES (2017).

BROUSSARD, Camille. Teaching with Technology: Is the Pedagogical Fulcrum Shifting? 53 NEW YORK LAW SCHOOL LAW REVIEW 903 (2009).

BROWN, Peter C. et al. Make It Stick:The Science Of Successful Learning 106-108 (2014).

BULLEN, Mark et al. Digital Learners in Higher Education: Generation is Not the Issue, 37 CANADIAN JOURNAL OF LEARNING ANDTECHNOLOGY / LA REVUE CANADIENNE DE L'APPRENTISSAGE ET DE LA TECHNOLOGIE 1 (2011), https: / /www.learntechlib.org/p/42755/ (visitado por última vez en 5 ago., 2019).

CARON, Paul L. Teaching with Technology in the 21st Century Law School Classroom 3 (2006), https:// papers.ssrn.com/abstract $=896906$ (visitado por última vez en 30 jul, 2019).

CARON, Paul L.; GELY, Rafael. Taking Back the Law School Classroom: Using Technology to Foster Active Student Learning Pedagogy, 54 J. LEGAL EDUC. 551 (2004). 
CHAKRABORTY, Saikat et. al. Education as an instrument of social change and enhancing teaching learning process with the help of technological development (2018), https: / www.researchgate.net/publication/ 325143953_Education_as_an_instrument_of_social_change_and_enhancing_teaching-learning_process_ with_the_help_of_technological_development.

CICERO, Nidia. Innovar la enseñanza del derecho. ¿Solo se trata de tecnologías de la información y comunicación? 5 REVISTA PEDAGÓGICA UNIVERSITARIAY DIDÁCTICA DEL DERECHO 91 (2018).

COMUNIDAD VIRTUAL, Facultad de Derecho, Universidad Externado, Los abogados y la tecnología, Universidad Externado de Colombia (2018), https: / / www.uexternado.edu.co/Derecho/los-abogadosy-la-tecnologia/ (visitado por última vez en 8 ago., 2019).

CORREAS, Carlos-Ignacio Massini. Filosofía del derecho I. El derecho y los derechos humanos. Buenos Aires, ABELEDO PERROT (1994).

COURAGE, Mary L. et al. Growing up multitasking: the costs and benefits for cognitive development, 35 DEVELOPMENTAL REVIEW 5 (2015).

CUBAN, Larry. Teachers And Machines:The Classroom Use Of Technology Since 1920 (1986).

CUNningham, Lawrence A. Digital Evolution in Law School Course Books, in Legal Education in The Digital Age 81 (Edward Rubin ed., 2012).

DAVIS, Hank. Caveman Logic:The persistence of primitive thinking in a modern world (2009).

DEGROFF, Eric A. Training Tomorrow's Lawyers:What Empirical Research Can Tell us about the Effect of Law School Pedagogy on Law Student Learning Styles, 36 S. ILL. U. L.J. 251 (2011).

DREYFUS, Hubert. Heidegger on Gaining a Free Relation to Technology, in READINGS IN THE PHILOSOPHY OF TECHNOLOGY 25 (David M. Kaplan ed., 2. ed. 2009).

DREYFUS, Hubert. Heidegger on the Connection between Nihilism, Art, Technology and Politics, in THE CAMBRIDGE COMPANION TO HEIDEGGER 345 (Charles B. Guignon ed., 2. ed. 2006).

EASTON, Catherine. An Examination of Clicker Technology Use in Legal Education, JILT (2009), https: / / warwick.ac.uk/fac/soc/law/elj/jilt/2009_3/easton.

EBO, Bosah. Cyberghetto or cybertopia? Race, Class and Gender on the Internet, PRAEGER (1998). 
EICKS, Jeannette. Educating Superior Legal Professional: Successful Modern Curricula Join Law and Technology 5.06 (2012), https: / / papers.ssrn.com/abstract=2465977 (visitado por última vez en 9 ago., 2019).

FARRELL, Lindsey; HURT, Andrew C. Training the Millennial Generation: Implications for Organizational Climate, v. 12, n. 1, JOURNAL OF ORGANIZATIONAL LEARNING AND LEADERSHIP (Primavera 2014).

FEENBERG, Andrew. QUESTIONING TECHNOLOGY (1999).

FEENBERG, Andrew. Constructivism and Technology Critique: Replies to Critics, 43 INQUIRY (2000a).

FEENBERG, Andrew. The Ontic and the Ontological in Heidegger's Philosophy of Technology: Response to Thomson, 43 INQUIRY (2000b).

FITZSIMONS, Patrick. Enframing education, in HEIDEGGER, EDUCATION, AND MODERNITY (Michael Peters ed., 2010).

FOER, Joshua. Moonwalking with Einstein:The Art and Science of Remembering Everything (2011).

FRAND, Jason. The Information Age Mindset: Changes in Students and Implications for Higher Education, EDUCAUSE REV., sept.-oct. 2000, https://er.educause.edu/-/media/files/article-downloads / erm0051.pdf.

FRIEDMAN, Ron. The Future Law Office: Going Virtual, 30 LAW PRAC. MGMT. 30 (2004).

GALANTER, Marc; HENDERSON, William D. The Elastic Tournament:A Second Transformation of the Big Law Firm, 60 STAN. L. REV. 1867 (2007).

GALLAGHER, Winifred. Rapt:Attention and the Focused Life (2010).

GALVES, Fred. Will Video Kill the Radio Star -Visual Learning and the Use of Display Technology in the Law School Classroom, 2004 U. ILL. J.L. TECH. \& POL'Y 195 (2004).

GERDY, Kristin B.; WISE, Jane H.; CRAIG, Alison. Expanding Our Classroom Walls: Enhancing Teaching and Learning through Technology, 11 LEGAL WRITING: J. LEGAL WRITING INST. 263 (2005).

GLENN, David. Divided Attention, CHRON. HIGHER EDUC. (28 fev. 2010), http: //chronicle.com/ article/Scholars-Turn-Their-Attention/63746/ [http: / / perma.cc/5V22-KZ6V]. 
GOLDMAN, Pearl. Legal Education and Technology:An Annotated Bibliography, 93 Law Libr. J. 423-468 (2001).

GOLDMAN, Pearl. Legal Education and Technology II:An Annotated Bibliography, 100 Law Libr. J. 415 (2008).

GRANAT, Richard; LAURITSEN, Marc. The Many Faces of E-Lawyering, 30 LAW PRAC. MGMT. 36 (2004).

GRISDELA, Margaret. s.f. Overview of the U.S. Legal Market, HG Legal Resources, https://www.hg.org/ marketing-us-market.html (visitado por última vez en 8 ago., 2019).

HARRIS, Nikos. The Risks of Technology in the Law Classroom: Why the Next Great Development in Legal Education Might Be Going Low-Tech, 51 U.B.C. L. REV. 773 (2018).

HARVARD LAW SCHOOL, Programming for Lawyers, HARVARD LAW SCHOOL (2017), https: / / hls.harvard.edu/academics / curriculum/catalog/default.aspx?o $=71516$ (visitado por última vez en 11 ago., 2019).

HAUN, Daniel. Repetition, Availability and Truth, Is the Internet Changing theWay You Think? The Net's Impact on Our Minds and Future 293, JOHN BROCKMAN ed. (2011).

HEIDEGGER, Martin. The question concerning technology, in THE QUESTION CONCERNING TECHNOLOGY AND OTHER ESSAYS 3 (William Lovitt tran., 1977).

HELSPER, Ellen J.; EYNON, Rebecca. Digital Natives:Where Is the Evidence? 36 BRIT. EDUC. RES. J. (2010).

HOWE, Neil; STRAUSS, William. Generations:The History of America's Future, 1548 to 2069 (1991).

HUME, David. Treatise of Human Nature, Libro III, Parte I, Sección I, in THE PHILOSOPHICAL WORKS, v. 2, s.f.

JACOBSON, M. H. Sam. Paying Attention or Fatally Distracted: Concentration, Memory, and Multi-Tasking in a Multi-Media World, 16 J. LEGAL WRITING INST. 419 (2010).

JOHNSON, Stephen M. www.lawschool.edu: Legal Education in the Digital Age, 2000Wis. L. Rev. 85 (2000).

JOHnSON, Stephen. Teaching for Tomorrow: Utilizing Technology to Implement the Reforms of MacCrate, Carnegie, and Best Practices, 92 NEBRASKA LAW REVIEW 46 (2013).

JONES, Chris et al. Net generation or Digital Natives: Is there a distinct new generation entering university? 54 COMPUTERS \& EDUCATION (2010). 
JONES, Chris; SHAO, Binhui. The Net Generation and Digital Natives: Implications for Higher Education, HIGHER EDUCATION ACADEMY (2011).

KAHNEMAN, Daniel. Thinking, Fast and Slow (2011).

KATZ, Martin J. Facilitating Better Law Teaching - Now The 2012 Randolph W. Thrower Symposium: Innovation for the Modern Era: Law, Policy, and Legal Practice in a Changing World, 62 EMORY L.J. $823(2012)$.

KELLNER, Douglas. New Technologies/New Literacies: Reconstructing education for the new millennium, 11 TEACHING EDUCATION 245 (2000).

KIMBRO, Stephanie. Receiving a Digital Legal Education | Lawyerist, LAWYERIST.COM (2010), https:/ / lawyerist.com/receiving-a-digital-legal-education/ (visitado por última vez en 9 ago., 2019).

LAMBEIR, Bert. Comfortably Numb in the Digital Era: Man's Being as Standing-reserve or Dwelling silently, in HEIDEGGER, EDUCATION, AND MODERNITY (Michael Peters ed., 2010).

LARSON, Joni. Turning the Tables: Is It Time for Professors to Stop Fighting the Presence of Students'Technology in the Classroom and Instead Use It to Enhance Student Learning? 43 N. KY. L. REV. 231 (2016).

LASSO, Rogelio. From the Paper Chase to the Digital Chase:Technology and the Challenge of Teaching 21 st Century Law Students, 43 SANTA CLARA L. REV. 1 (2002).

LATRUP-PEDERSEN, Tom. Trends in Legal Education in the Learning Society — The Challenge of Information Technology, 9 INTERNATIONAL JOURNAL OFTHE LEGAL PROFESSION 165-186 (2002).

LEE, Jennifer; LIN, Lin; ROBERTSON, Tip. The impact of media multitasking on learning, 37 LEARNING, MEDIA AND TECHNOLOGY 94-104 (2012).

LEVITIN, Daniel J. The Organized Mind:Thinking straight in the age of information overload 16 (2014).

LEVY, James B. Teaching the Digital Caveman: Rethinking the Use of Classroom Technology in Law School, 19 CHAP. L. REV. 241 (2016).

LOHR, Steve. Where Non-Techies Can GetWith the Programming, THE NEWYORK TIMES, April 4, 2017, https: / / www.nytimes.com/2017/04/04/education/edlife/where-non-techies-computer-programmingcoding.html (visitado por última vez en 11 ago., 2019). 
LUI, Kelvin F. H.; WONG, Alan C. N. Does media multitasking always hurt? A positive correlation between multitasking and multisensory integration, 19 PSYCHON BULL REV 647-653 (2012).

MAHARG, Paul; MUNTJEWERFF, Antoinette J. Through a screen, darkly: Electronic legal education in Europe, 36 THE LAW TEACHER 307-332 (2002).

MARCUS, Richard L. The Electronic Lawyer Symposium: The Challenge of 2020: Preparing a Civil Justice Reform Agenda for the Coming Decade: Fourteenth Annual Clifford Symposium on Tort Law and Social Policy, 58 DEPAUL L. REV. 263 (2008).

MCGINNIS, John O.; PEARCE, Russell G. The Great Disruption: How Machine IntelligenceWill Transform the Role of Lawyers in the Delivery of Legal Services Col loquium:The Legal Profession's Monopoly on the Practice of Law, 82 FORDHAM L. REV. 3041 (2013).

MEDINA, John. Brain Rules: 12 Principles for Surviving and Thriving at Work, Home, and School (2008).

MOGHADDAM, Golnessa Galyani. Information technology and Gender Gap: Toward a global view, s.f., EMERALD GROUP PUBLISHING LIMITED, THE ELECTRONIC LIBRARY, v. 28, n. 5.

MOPPETT, Samantha. Control-Alt-Incomplete? Using Technology to Assess “Digital Natives”, 12 CHICAGOKENT JOURNAL OF INTELLECTUAL PROPERTY 77 (2013).

MORRIS, Jason. How programming can make the law more accessible | Jason Morris | TEDxUAlberta (2018), YOUTUBE, https: / / www.youtube.com/watch?v=d5Mt-Q9K7tU (visitado por última vez en 11 ago., 2019).

MURRAY, Kristen E. Let Them Use Laptops: Debunking the Assumptions Underlying the Debate over Laptops in the Classroom, 36 OKLA. CITY U. L. REV. 185 (2011).

NASS, Clifford. FRONTLINE, https:/ /www.pbs.org/wgbh/pages/frontline/digitalnation/interviews/ nass.html (visitado por última vez en 12 ago., 2019).

OHM, Paul. Computer Programming and the Law:A New Research Agenda, 54 VILL. L. REV. 117 (2009).

OLUBIYI, Ifeoluwa; OLANIYAN, Ayobami; ODIAKA, Ngozi. The Role of Technology in the Advancement of Legal Education and Practice in Nigeria, 2 (2015), https: / / www.researchgate.net/publication/280566346_ The_Role_of_Technology_in_the_Advancement_of_Legal_Education_and_Practice_in_Nigeria.

O’NEIL, Megan. Confronting the Myth of the 'Digital Native,' CHRON. HIGHER EDUC. (21 abr., 2014), http: / / chronicle.com/article/Confronting-the-Myth-of-the/145949/ [http:/ / perma.cc/S5L8-CN8Z]. 
PAGEL, Mark. Brain Candy \& Bad Mathematics, in IS THE INTERNET CHANGING THE WAY YOU THINK? THE NET'S IMPACT ON OUR MINDS AND FUTURE, John Brockman (ed.) (2011).

PALFREY, John. Smarter Law School Casebooks, in LEGAL EDUCATION IN THE DIGITAL AGE, Edward Rubin (ed.) (2012).

PAUL, Gregory. Hell if I Know, in IS THE INTERNET CHANGING THE WAY YOU THINK? THE NET’S IMPACT ON OUR MINDS AND FUTURE, John Brockman (ed.) (2011).

PINKER, Steven. How the MindWorks (1997).

PINKER, Steven. Not at All, in IS THE INTERNET CHANGING THE WAY YOU THINK? THE NET'S IMPACT ON OUR MINDS AND FUTURE, John Brockman (ed.) (2011).

PISTONE, Michele. Law Schools and Technology:WhereWe Are andWhereWe Are Heading Igniting Law Teaching, 64 J. LEGAL EDUC. 586 (2014).

PIVOVAROV, Valentin. 713\% Growth: Legal Tech Set An Investment Record In 2018, FORBES, https:// www.forbes.com/sites/valentinpivovarov/2019/01/15/legaltechinvestment2018/ (visitado por última vez en 9 ago., 2019).

PRENSKY, Marc. Digital Natives, Digital Immigrants Part 1, 9 ON THE HORIZON 1 (2001).

PRIEST, Stephen. The British Empiricists (2. ed. 2007).

RATEY, John J. A User's Guide to The Brain (2001).

RAVIZZA, Susan M. et al. Non-academic Internet Use in the Classroom Is Negatively Related to Classroom Learning Regardless of Intellectual Ability, 78 COMPUTERS \& EDUC. (2014).

RICHTEL, Matt. Technology Changing How Students Learn, Teachers Say, N.Y. TIMES, en A18 (1 nov., 2012), https:/ / www.nytimes.com/2012/11/01/education/technology-is-changing-how-studentslearn-teachers-say.html.

RIQUELME, Rodrigo. LegalTech: el valor y los retos de la tecnologia en el mundo del derecho (2019), EL ECONOMISTA, https: / / www.eleconomista.com.mx/tecnologia/Legal-Tech-el-valor-y-los-retos-dela-tecnologia-en-el-mundo-del-derecho-20190228-0148.html (visitado por última vez en 8 ago., 2019).

RUBIN, Edward L. (ed.). Legal Education in the Digital Age (2012). 
SAW, Yenée. Putting technology in its Place:Are Law Schools the Right Place to Teach Coding? LEGAL INSIGHT (2018), http://insight.thomsonreuters.com.au/posts/law-schools-teach-coding (visitado por última vez en 11 ago, 2019).

SCHANK, Roger. Teaching Minds: How Cognitive Science Can Save Our Schools (2011).

SCHANK, Roger. The thinking Process Hasn't Changed in 50,000 Years, EDGE, https: / / perma.cc/SJV9HEVH (visitado por última vez en 8 ago., 2019).

SHERWIN, Richard K.; FEIGENSON, Neal; SPIESEL, Christina. Law in the Digital Age: How Visual Communication Technologies are Transforming the Practice, Theory, and Teaching of Law, 12 B.U. J. SCI. \& TECH. L. 227 (2006).

SCHILTZ, Patrick J. On Being a Happy, Healthy, and Ethical Member of an Unhappy, Unhealthy, and Unethical Profession Symposium: Attorney Well-Being in Large Firms: Choices Facing Young Lawyers, 52 VAND. L. REV. 871 (1999).

SHELL, Duane F. et al. The Unified Learning Model: How Motivational, Cognitive, And Neurobiological Sciences Inform Best Teaching Practices (2010).

SIECKMANN, Jan R. La Sociología del derecho en la formación jurídica. ACADEMIA. REVISTA SOBRE ENSEÑANZA DEL DERECHO, n. 12, p. 117-133 (2008).

SITES, Brian. The Influence of Algorithms:The Importance of Tracking Technology as Legal Educators, 23 LAW TCHR. 21 (2016).

SK, Md Esahaque. Education as an instrument of social change, INTERNATIONAL RESEARCH JOURNAL OF MULTIDICSIPLINARY STUDIES, V. 3, I. 6 (jun. 2017).

SKIBA, Diane J.; BARTON, Amy J. AdaptingYour Teaching to Accommodate the Net Generation of Learners, THE ONLINE JOURNAL OF ISSUES IN NURSING, v. 11 (2006).

SLOMANSON, William R. Blended Learning:A Flipped Classroom Experiment, 64 J. LEGAL EDUC. 93 (2014).

STANDISH, Paul. Only Connect: Computer Literacy from Heidegger to Cyberfeminism, 49 EDUCATIONAL THEORY 417-435 (1999).

STEELE, Thomas. The MacCrate Report: Its Impact on Education in Law Firm Management, 23 Pace Law Review 613 (2003). 
STEINER, George. HEIDEGGER 211-212 (4. ed. 2013).

SYLWESTER, Robert. A Celebration of Neurons: An Educator's Guide to The Human Brain (1995).

TENINBAUM, Gebe. Reimagining Innovation In Legal Education (2019), https://abovethelaw.com/ career-files/reimagining-innovation-in-legal-education/ (visitado por última vez en 30 jul., 2019).

THE IRISHTIMES CONTENT STUDIO. Technology drives change and creates new solutions for legal clients, THE IRISH TIMES (2019), https://www.irishtimes.com/special-reports/innovation-inlaw/technology-driveschange-and-creates-new-solutions-for-legal-clients-1.3769572 (visitado por última vez en 11 ago., 2019).

THOMPSON, Penny. The Digital Natives as Learners: Technology Use Patterns and Approaches to Learning, 65 COMPUTERS \& EDUC. (2013).

THOMSON, Iain D. HEIDEGGER ON ONTOTHEOLOGY: TECHNOLOGY AND THE POLITICS OF EDUCATION 53 (2005).

THOMSON, Iain. Ontotheology? Understanding Heidegger's Destruktion of Metaphysics, 8 INTERNATIONAL JOURNAL OF PHILOSOPHICAL STUDIES 297 (2000).

THREEDY, Debora L.; DEWALD, Aaron. Re-Conceptualizing Doctrinal Teaching: Blending Online Videos with in-Class Problem-Solving, 64 J. LEGAL EDUC. 605 (2015).

USC GOULD SCHOOL OF LAW. The Rise of Virtual Law Practices and "E-Lawyering”, Online International LLM Degree Program (2017), https: / / onlinellm.usc.edu/blog/elawyering-and-virtual-law-practices / (visitado por última vez en 7 ago., 2019).

TROKSA, Lauren. The Study of Generations:A Timeless Notion within a Contemporary Context, UNIVERSITY OF COLORADO, CU SCHOLAR (Primavera, 2016).

WADDINGTON, David I. A Field Guide to Heidegger: Understanding 'The Question Concerning Technology', v. 37, n. 4 EDUCATIONAL PHILOSOPHY AND THEORY 567 (2005).

WALLACE, James E. Philosophy and the future law school curriculum, 44 DENV L.J. 24 (1967).

WANG, Shiang-Kwei et al. An Investigation of Middle School Science Teachers and Students Use of Technology Inside and Outside of Classrooms: ConsideringWhether Digital Natives Are More Technology Savvy than Their Teachers, 62 EDUC. TECH. RES. \& DEV. (2014). 
WARNER, Richard; SOWLE, Stephen D; SADLER, Will. Teaching Law with Computers, 24 RUTGERS COMPUTER \& TECH. L.J. 107 (1998).

WARREN, Kay B.; BOURQUE, Susan C. Women, Technology and International Development Ideologies:Analyzing FeministVoices, in Gender at the Crossroads of Knowledge: Feminist Anthropology in the Postmodern Era, UNIVERSITY OF CALIFORNIA PRESS (1991).

WILLIS, Miguel. 8 Law Schools on Cutting Edge of Tech + Innovation, Innovative Law Student (2016), https: / / www.innovativelawstudent.com/2016/04/7-law-schools-cutting-edge-tech-innovation/ (visitado por última vez en 30 jul. 2019).

WOLF, Maryanne. Proust And the Squid:The Story and Science of The Reading B rain (2007).

ZARRETT, Nicole R.; MALANCHUK, Oksana. Who's Computing? Gender and Race Differences in Young Adults Decisions to Pursue and Information Technology Career, NEW DIRECTIONS FOR CHILD AND ADOLESCENT DEVELOPMENT, I. (2005).

\section{CómO CITAR ESTE ARTí́CULO:}

MALDONADO, Daniel Bonilla. Educación jurídica e innovación tecnológica: un ensayo crítico. Revista Direito GV, v. 16, n. 1, jan./abr. 2020, e1954. doi: http://dx. doi.org/10.1590/2317-6172201954.
Daniel Bonilla Maldonado

Profesor Titular, Facultad de Derecho, Universidad de LOS ANDES.

dbonillalauniandes.edu.co 